\title{
THE LOWER TAIL OF THE HALF-SPACE KPZ EQUATION
}

\author{
YUJIN H. KIM
}

\begin{abstract}
We establish the first tight bound on the lower tail probability of the half-space KPZ equation with Neumann boundary parameter $A=-1 / 2$ and narrow-wedge initial data. When the tail depth is of order $T^{2 / 3}$, the lower bound demonstrates a crossover between a regime of superexponential decay with exponent $\frac{5}{2}$ (and leading pre-factor $\frac{2}{15 \pi} T^{1 / 3}$ ) and a regime with exponent 3 (and leading pre-factor $\frac{1}{24}$ ); the upper bound demonstrates a crossover between a regime with exponent $\frac{3}{2}$ (and arbitrarily small pre-factor) and a regime with exponent 3 (and leading pre-factor $\frac{1}{24}$ ). We show that, given a crude leading-order asymptotic in the Stokes region (Definition 1.8, first defined in (Duke Math J., [Bot17])) for the Ablowitz-Segur solution to the Painlevé II equation, the upper bound on the lower tail probability can be improved to demonstrate the same crossover as the lower bound. We also establish novel bounds on the large deviations of the GOE point process.
\end{abstract}

\section{CONTEnts}

1. Introduction 2

1.1. The half-space KPZ equation with Neumann boundary conditions 2

1.2. Fluctuations of the GOE point process 5

1.3. Asymptotics of the Ablowitz-Segur solution to the Painlevé II equation 8

1.4. Main results on the GOE Point Process 9

1.5. Outline 10

Acknowledgements $\quad 10$

2. Proof of the main theorem 11

2.1. Proof of Theorem 1.4 11

2.2. Proof of Proposition 2.1 12

3. The GOE point process 12

3.1. First notions $\quad 13$

3.2. The $\beta$ stochastic Airy operator $\quad 15$

4. The cumulant generating function for $\chi^{\mathrm{GOE}} \quad 17$

4.1. The thinned GOE point process and the Painlevé II equation 17

4.2. Fredholm Pfaffians 18

4.3. Proofs of Theorems 1.7 and $1.10 \quad 19$

4.4. Proof of Lemma $1.9 \quad 20$

5. Proof of Theorem $1.11 \quad 22$

6. Proof of Theorem $1.12 \quad 22$

7. Proof of Proposition 2.2 26

7.1. Proof of the upper bound, equations (2.11) and (2.12) 26

7.2. Proof of the lower bound, equation (2.10) 30

References $\quad 38$

2010 Mathematics Subject Classification. Primary: 60H15. Secondary: 60B20, 45M05, 60F10, 60G55, $60 \mathrm{H} 25$.

Key words and phrases. (Half-space) Kardar-Parisi-Zhang equation, Pfaffian point processes, GOE ensemble, large deviations, stochastic Airy operator, Ablowitz-Segur Solution to Painlevé II. 


\section{INTRODUCTION}

The Kardar-Parisi-Zhang (KPZ) equation is formally given by

$$
\partial_{T} H(T, X)=\frac{1}{2} \partial_{X}^{2} H(T, X)+\frac{1}{2}\left(\partial_{X} H(T, X)\right)^{2}+\xi(T, X),
$$

where $T \geq 0, X \in \mathbb{R}$, and $\xi$ is Gaussian space-time white noise with covariance $\mathbb{E}[\xi(T, X) \xi(S, Y)]=$ $\delta(T-S) \delta(X-Y)$. A physically relevant notion of solution to this equation is given by the Cole-Hopf solution to the KPZ equation with narrow-wedge initial data

$$
H(T, X):=\log Z(T, X), \quad \text { with } Z(0, X)=\delta_{0}(X),
$$

where $Z$ solves the $(1+1)$ d stochastic heat equation (SHE) with multiplicative space-time white noise

$$
\partial_{T} Z(T, X)=\frac{1}{2} \partial_{X}^{2} Z(T, X)+Z(T, X) \xi(T, X) .
$$

The well-definedness of (1.2) is given by the work of [Mue91] establishing almost-sure positivity of $Z$ for a wide class of initial data (including delta initial data).

The KPZ equation is a paradigmatic model in a class of models, known as the KPZ universality class, whose long-time limit is the KPZ fixed point. While this universality class is not strictly defined, all models in this class should share specific salient features. The KPZ equation itself has been shown to govern the long-time limits under weak asymmetric scaling of many other models in the universality class. The notes and surveys [Fer10], [Cor12], [Cor14] [QS15], [Sas16], [Tak18], and [Zyg18] provide further reading on various aspects of the KPZ universality class.

Just as in the full-space case, the half-space KPZ equation with Neumann boundary conditions plays a significant role within the half-space KPZ universality class. Mathematical analysis of the half-space analogues of models believed to lie in the KPZ universality class began with the work of [BR01, IS04], both of which consider variants of half-space TASEP. For a recent result relating to half-space TASEP, see [BBCS18]. Progress has been especially fruitful in the case of ASEP. [CS18] established convergence of the height function of half-space ASEP under weakly asymmetric scaling to the half-space KPZ equation with Neumann boundary parameter $A \geq 0$. Following this result, [BBCW18] established an exact one-point distribution formula for half-space ASEP with $A=-1 / 2$, and [Par19a] was able to extend the work of [CS18] to show convergence to the half-space KPZ equation for all real $A$. See, for instance, [KLD18a], [Wu18], [KLD20], [BBC20], [BKLD20], [Lin20], and $[\mathrm{BFO} 20]$ for additional results in the half-space $\mathrm{KPZ}$ universality class.

We now describe the half-space KPZ equation in detail.

1.1. The half-space KPZ equation with Neumann boundary conditions. This paper seeks to establish bounds on the lower tail of the half-space KPZ equation with Neumann boundary condition, an object which we presently define.

Definition 1.1 (Mild solution to the half-space SHE, half-space KPZ). We say $\mathscr{Z}(T, X)$ is a mild solution to the SHE given in (1.3) on $\mathbb{R}_{+}$with delta initial data at the origin and Robin boundary condition with parameter $A \in \mathbb{R}$

$$
\left.\partial_{X} \mathscr{Z}(T, X)\right|_{X=0}=A \mathscr{Z}(T, 0), \forall T>0
$$

if $\mathscr{Z}(T, \cdot)$ is adapted to the filtration given by $\sigma\left(\mathscr{Z}(0, \cdot),\left.W\right|_{[0, T]}\right)$ and the following Duhamel-form identity is satisfied

$$
\mathscr{Z}(T, X)=\int_{0}^{\infty} \mathscr{P}_{T}^{R}(X, Y) Z(0, Y) d Y
$$




$$
+\int_{0}^{T} \int_{0}^{\infty} \mathscr{P}_{T-S}^{R}(X, Y) Z(S, Y) \xi(S, Y) d W_{S}(d Y)
$$

for all $T>0$ and $X>0$. Here, the last integral is Itô with respect to the cylindrical Wiener process $W$, and $\mathscr{P}^{R}$ is the heat kernel on $[0, \infty)$, i.e., the fundamental solution to the heat equation on $[0, \infty)$, satisfying the Robin boundary condition

$$
\left.\partial_{X} \mathscr{P}_{T}^{R}(X, Y)\right|_{X=0}=A \mathscr{P}_{T}^{R}(0, Y), \forall T>0, Y>0 .
$$

The Hopf-Cole solution to the half-space KPZ equation with Neumann boundary parameter $A$ is then defined to be $H=\log \mathscr{Z}$.

[Par19a, Proposition 4.2] establishes the existence, uniqueness, and almost-sure positivity of $\mathscr{Z}(T, \cdot)$ for all $A \in \mathbb{R}$, which makes the Hopf-Cole solution to the half-space KPZ equation with Neumann boundary condition $A \in \mathbb{R}$ well-defined.

Our paper establishes tight bounds on the lower tail probability of $H(T, 0)$, that is, the probability that $\mathscr{Z}(T, 0)$ is very close to 0 , or equivalently, that $H(T, 0)$ is very negative, for the critical boundary parameter $A=-1 / 2$. Our result builds on the method used by [CG20] to find analogous bounds for the full-space KPZ lower tail.

We now explain the choice of boundary parameter $A=-1 / 2$. For this particular boundary parameter, [Par19a, Theorem 1.1] established Tracy-Widom GOE fluctuations at the origin.

Proposition 1.2 ([Par19a, Theorem 1.3]). Let $H(T, X)$ be the solution to the half-space KPZ equation with inhomogeneous Neumann boundary parameter $A=-1 / 2$ and narrow-wedge initial data (which corresponds to $\delta_{0}$ initial data for the SHE). Then the following weak convergence result holds

$$
\lim _{T \rightarrow \infty} \mathbb{P}\left(\Upsilon_{T} \leq s\right)=F_{\mathrm{GOE}}(s), \quad \text { where } \Upsilon_{T}:=\frac{H(2 T, 0)+\frac{T}{12}}{T^{1 / 3}}
$$

Here, $F_{\mathrm{GOE}}(s)$ is the Tracy-Widom GOE fluctuations [TW96], and $\Upsilon_{T}$ is the solution to the KPZ equation after centering and re-scaling.

For other choices of $A$, establishing the limiting fluctuations of $\Upsilon_{T}$ has been elusive, and thus establishing lower tail bounds in these regimes seems at the moment unfeasible. [Par19a, Conjecture 1.2] gives a conjecture establishing exactly two more regimes of distinct fluctuations: $A<-1 / 2$, with Gaussian fluctuations, and $A>-1 / 2$, with Tracy-Widom GSE distribution [TW96]. [Par19a, Section 1.3] gives a heuristic argument for the Gaussianity of the $A<-1 / 2$ regime; see also [Par19b]. [GLD12, BBC16, KLD20] provides strong evidence towards the conjectured $A>-1 / 2$ regime, though we emphasize that no part of this conjecture has been rigorously established.

On the other hand, for $A=-1 / 2$, we have access to Proposition 1.3 , which provides the starting point for our analysis.

Proposition 1.3 ([Par19a]). Let $H(T, X)$ denote the solution to the half-space KPZ equation on $[0, \infty)$ with Neumann boundary parameter $A=-1 / 2$ and narrow-wedge initial data. Then for $u>0$,

$$
\mathbb{E}_{\mathrm{SHE}}\left[\exp \left(-u \exp \left(H(2 T, 0)+\frac{T}{12}\right)\right)\right]=\mathbb{E}_{\mathrm{GOE}}\left[\prod_{k=1}^{\infty} \frac{1}{\sqrt{1+4 u \exp \left(T^{1 / 3} \mathrm{a}_{k}\right)}}\right] .
$$

Here, the $\left(\mathrm{a}_{1}>\mathrm{a}_{2}>\ldots\right)$ form the GOE point process (defined in Section 3.1). 
Taking $u:=\frac{1}{4} \exp \left(T^{1 / 3} s\right)$ in (1.9) and recalling $\Upsilon_{T}$ from (1.8), we obtain

$$
\mathbb{E}_{\mathrm{SHE}}\left[\exp \left(-\frac{1}{4} \exp \left(T^{1 / 3}\left(\Upsilon_{T}+s\right)\right)\right)\right]=\mathbb{E}_{\mathrm{GOE}}\left[\prod_{k=1}^{\infty} \frac{1}{\sqrt{1+\exp \left(T^{1 / 3}\left(\mathrm{a}_{k}+s\right)\right)}}\right]
$$

Note that the function $\exp (-\exp (x))$ is an approximate of the indicator function $\mathbb{1}(x \leq 0)$, and so the integrand of the left-hand side of (1.10) approximates $\mathbb{P}\left(\Upsilon_{T}+s \leq 0\right)$ for large $s$. This heuristic is made rigorous in Section 2.1. Proposition 1.3 was conjectured in [BBCW18, Theorem 7.6], which proves the analogous formula for the height function of half-space ASEP and computes asymptotics which were expected to lead to the above result on the KPZ equation. Combining their result with [Par19a, Theorem 1.2] yields Proposition 1.3.

We our now ready to state our main result, Theorem 1.4, which establishes upper and lower bounds on the lower tail probability $\mathbb{P}\left(\Upsilon_{T} \leq-s\right)$ for large but fixed times $T>0$.

Theorem 1.4. Let $\Upsilon_{T}$ denote the solution to the half-space $K P Z$ equation with Neumann boundary parameter $A=-1 / 2$ and narrow-wedge initial data, centered and re-scaled as in (1.8). Fix any $\eta>0, \varepsilon \in(0,1 / 3), \delta \in(0,1 / 4)$, and $T_{0}>0$. There exist positive constants $S:=S\left(\eta, \varepsilon, \delta, T_{0}\right)$, $C:=C\left(T_{0}\right), K_{1}:=K_{1}\left(\varepsilon, \delta, T_{0}\right)$, and $K_{2}:=K_{2}\left(T_{0}\right)$ such that for all $s \geq S$ and $T \geq T_{0}$, we have

$$
\mathbb{P}\left(\Upsilon_{T} \leq-s\right) \geq e^{-\frac{2(1+C \varepsilon)}{15 \pi} T^{1 / 3} s^{5 / 2}}+e^{-K_{2} s^{3}},
$$

and

$$
\mathbb{P}\left(\Upsilon_{T} \leq-s\right) \leq e^{-\frac{2(1-C \varepsilon)}{15 \pi} T^{1 / 3} s^{5 / 2}}+e^{-\frac{\varepsilon}{2} s T^{1 / 3}-\eta s^{3 / 2}}+e^{-\frac{1-C \varepsilon}{24} s^{3}}
$$

Assuming Conjecture 1, we have the stronger

$$
\mathbb{P}\left(\Upsilon_{T} \leq-s\right) \leq e^{-\frac{2(1-C \varepsilon)}{15 \pi} T^{1 / 3} s^{5 / 2}}+e^{-\frac{\varepsilon}{2} s T^{1 / 3}-K_{1} s^{3-\delta}}+e^{-\frac{1-C \varepsilon}{24} s^{3}} .
$$

Conjecture 1 has a rather technical statement regarding the leading-order asymptotics of AblowitzSegur solution $u_{\mathrm{AS}}(x ; \gamma)$ to the Painlevé II equation in a certain region, named the Stokes region. Its openness is due to the difficulty of a certain Riemann-Hilbert problem. One major goal of this article is to highlight the direct connection between leading-order asymptotics of $u_{\mathrm{AS}}(x ; \gamma)$ in the Stokes region and the lower-tail of the KPZ equation, in hopes of motivating further study of the Stokes region. For the sake of a more stream-lined discussion of Theorem 1.4 and its proof, we postpone a detailed discussion of Conjecture 1 and the Painlevé II equation to Section 1.3. The proof of Theorem 1.4 is given in Section 2.1. We note that (1.12) and (1.13) differ only in the second term of each.

We can see Theorem 1.4 displays three distinct regions of decay as follows. First, note that Proposition 1.2 implies that, as $T \rightarrow \infty, \mathbb{P}\left(\Upsilon_{T}<-s\right)$ should decay according to $F_{\mathrm{GOE}}(-s)$, which is approximately $\exp \left(-\frac{1}{24} s^{3}\right)$ for large $s$ (see Proposition 7.1). This cubic decay is exhibited in the last terms of (1.11)-(1.13). Note that in the range $T^{2 / 3} \gg s \gg 0$, either the second or the third term of (1.13) dominates; in (1.11), the second term dominates (though in the lower bound (1.11), the prefactor of the cubic exponent is not explicit). When $T \rightarrow \infty$, the third term of (1.13) dominates and thus recovers the cubic decay of the $F_{\mathrm{GOE}}$ tail. On the other hand, in the "short time deep tail" region $s \gg T^{2 / 3}$, the first term of both (1.13) and (1.11) dominates; however, in (1.12), the second term dominates the first term in all regions. The $5 / 2$ exponent and the $\frac{2}{15 \pi}$ prefactor for this region were first observed in [KLD18b]. The crossover from $5 / 2$ to cubic exponent that occurs when $s$ is of order $T^{2 / 3}$ can be understood in terms of large deviations: as $T \rightarrow \infty$, the crossover is exhibited by the large deviation rate function for the half-space KPZ equation, which has speed $T^{2}$. In the full-space case, this crossover was first predicted by [SMP17], which also contains the first prediction of the full-space rate function; [CGK ${ }^{+} 18, \mathrm{KLD}_{18 \mathrm{a}}$, KLDP18] each provide alternative methods of computing this rate function. In particular, $\left[\mathrm{CGK}^{+} 18\right]$ showed that the half-space rate 
function is simply one-half that of the full space. The rate functions for both the full and half-space case were finally rigorously established by [Tsa18]. Just over a year after the posting of this paper, the preprint [Zho20] obtained sharper upper and lower bounds than in Theorem 1.4 by proving large deviation bounds for the Airy point process. In particular, their upper-bound on the lower tail probability is given by $e^{-\frac{2(1-C \varepsilon)}{15 \pi} T^{1 / 3} s^{5 / 2}}+e^{-\frac{\varepsilon}{2} s T^{1 / 3}-K s^{3}}+e^{-\frac{1-C \varepsilon}{24} s^{3}}$, so that the aforementioned crossover from exponent $5 / 2$ to 3 is attained. Large deviation bounds for the Airy point process were originally (non-rigorously) derived by $\left[\mathrm{CGK}^{+} 18\right]$ using Coulomb gas heuristics.

The techniques used to prove Theorem 1.4 are heavily inspired by the work of [CG20] on the lower tail of the full-space KPZ equation. Their work starts with the full-space KPZ analog to (1.9), which was established in [BG16], where the full-space KPZ equation is related to a multiplicative functional of the Airy (GUE) point process by manipulations of an exact formula for the one-point distribution of SHE with delta initial data. This one-point distribution formula was simultaneously and independently computed in [ACQ11, SS10, CLDR10, Dot10] and rigorously proved in [ACQ11]. In [CG20], the formula of [BG16] was manipulated to yield tight bounds on the lower tail of the fullspace KPZ equation; however, in order to do this, [CG20] first establishes appropriate control on the fluctuations of the GUE point process. Their work strongly suggests that a careful manipulation of (1.10) would similarly yield tight bounds on the lower tail of the half-space KPZ equation, given analogous control on the GOE point process; indeed, this is the approach taken in the current article. We now outline our approach to studying the GOE point process and the methods used therein.

1.2. Fluctuations of the GOE point process. In Section 3.1, we define the GOE point process and describe its key properties as a simple Pfaffian point process (also defined in that section). The estimates on the GOE point process needed in this article pertain to (1) controlling the locations of individual GOE points, and (2) controlling the number of GOE points within intervals.

Towards (1), we detail in Section 3.2 the well-studied connection between the (stochastic) Airy operator (SAO) and the GOE points, and describe the relevant known results (Propositions 3.23.4). In particular, the seminal work of [RRV11] (Proposition 3.2) gives an equivalence in distribution between the eigenvalues of the $\beta=1 \mathrm{SAO}$ and the GOE points, while [CG20, Proposition 4.5] (Proposition 3.3 below) establishes uniform control on the deviations of the (random) SAO eigenvalues from deterministic locations given by the eigenvalues $\left(\lambda_{k}\right)$ of the (deterministic) Airy operator. Theorem 1.5 below is then simply the combination of Proposition 3.2 and Proposition 3.3.

Theorem 1.5. For $\varepsilon \in(0,1)$, let $C_{\varepsilon}^{\mathrm{GOE}}$ be the smallest real number such that, for all $k \geq 1$,

$$
(1-\varepsilon) \lambda_{k}-C_{\varepsilon}^{\mathrm{GOE}} \leq-\mathrm{a}_{k} \leq(1+\varepsilon) \lambda_{k}+C_{\varepsilon}^{\mathrm{GOE}},
$$

where $\mathrm{a}_{k}$ is the $k^{\text {th }}$ largest point of the GOE point process and $\lambda_{k}$ is the $k^{\text {th }}$ smallest eigenvalue of the Airy operator. Then, for all $\varepsilon, \delta \in(0,1)$, there exist constants $S_{0}:=S_{0}(\varepsilon, \delta)$ and $\kappa:=\kappa(\varepsilon, \delta)$ such that, for all $s \geq S_{0}$,

$$
\mathbb{P}\left(C_{\varepsilon}^{\mathrm{GOE}} \geq s\right) \leq \kappa \exp \left(-\kappa s^{1-\delta}\right)
$$

Theorem 1.5 establishes an upper bound on the probability that the $\mathrm{a}_{k}$ deviate away from the (deterministic) $\lambda_{k}$, uniformly in $k$. This is extremely helpful because we know what the $\lambda_{k}$ look like: Proposition 3.4 tells us that ${ }^{1} \lambda_{k} \sim\left(\frac{3 \pi}{2} k\right)^{2 / 3}$.

Towards (2), we define the counting function

$$
\chi^{\mathrm{GOE}}: \mathcal{B}(\mathbb{R}) \rightarrow \mathbb{Z}_{\geq 0}, \quad \chi^{\mathrm{GOE}}(B):=\#\left\{k: \mathrm{a}_{k} \in B\right\}, \forall B \in \mathcal{B}(\mathbb{R}),
$$

\footnotetext{
${ }^{1}$ Here, $f(k) \sim g(k)$ if they are asymptotically equivalent, i.e., $\lim _{k \rightarrow \infty} \frac{f(k)}{g(k)}=1$.
} 
where $\mathcal{B}(\mathbb{R})$ denotes the Borel $\sigma$-algebra of $\mathbb{R} . \quad \chi^{\mathrm{GOE}}(\cdot)$ is a non-negative integer-valued random measure on $(\mathbb{R}, \mathcal{B}(\mathbb{R}), \mu)$, where $\mu$ denotes the Lebesgue measure on $\mathbb{R}$, that, informally speaking, counts the number of GOE points in a Borel set $B$ - see Section 3.1 for a formal description. We will also refer to $\chi^{\mathrm{GOE}}$ as the GOE point process. The mean of $\chi^{\mathrm{GOE}}$ on intervals is given by Theorem 1.6 below, which is proved at the end of Section 3.1.

Theorem 1.6. Define the interval $\mathfrak{B}_{1}(s):=[-s, \infty)$. For any $s>0$, we have

$$
\mathbb{E}_{\mathrm{GOE}}\left[\chi^{\mathrm{GOE}}\left(\mathfrak{B}_{1}(s)\right)\right]=\frac{2}{3 \pi} s^{3 / 2}+D_{1}(s),
$$

where $\sup _{s>0}\left|D_{1}(s)\right|<\infty$.

We expect that this result and other statistics for $\chi^{\mathrm{GOE}}$ should be known; however, we were unable to find such results in the literature. Note that the leading-order term $s^{3 / 2}$ of (1.16) matches the leading-order term of the expectation of the GUE (or, Airy) point process $\chi^{\mathrm{Ai}}$ on $\mathfrak{B}_{1}(s)$, computed in [Sos00]. [Sos00] also computes the variance of and establishes a central limit theorem for $\chi^{\mathrm{Ai}}$.

In light of Theorem 1.6, we are interested in deviations of order $s^{3 / 2}$ of $\chi^{\mathrm{GOE}}$ on intervals of size $s$. The upper deviations result (Theorem 1.12, proved in Section 6) will actually follow from the results discussed in (1) and the lower deviations result (Theorem 1.11, proved in Section 5), and so we now turn our attention to the lower deviations. To introduce important related objects and motivate the results that follow, we begin with a preliminary computation of the lower deviations of $\chi^{\mathrm{GOE}}$. Recall from Theorem 1.6 the interval $\mathfrak{B}_{1}(s)$. For any $s \in \mathbb{R}$ and $v>0$, define

$$
F_{1}(s, v):=\mathbb{E}\left[\exp \left(-v \chi^{\mathrm{GOE}}\left(\mathfrak{B}_{1}(s)\right)\right)\right] .
$$

$F_{1}(s, v)$ is the cumulant generating function for $\chi^{\mathrm{GOE}}$. Now, for any positive $c, v$ and $s$, taking $f(x)=e^{-v x}$ in Markov's inequality and then applying Theorem 1.6 yields

$$
\begin{aligned}
& \mathbb{P}\left(\chi^{\mathrm{GOE}}\left(\mathfrak{B}_{1}(s)\right)-\mathbb{E}\left[\chi^{\mathrm{GOE}}\left(\mathfrak{B}_{1}(s)\right)\right] \leq-c s^{3 / 2}\right) \\
& \leq \exp \left(-c v s^{3 / 2}+v \mathbb{E}\left[\chi^{\mathrm{GOE}}\left(\mathfrak{B}_{s}\right)\right]\right) F_{1}(-s, v), \\
& =\exp \left(\left(\frac{2}{3 \pi}-c\right) v s^{3 / 2}+v D_{1}(s)\right) F_{1}(-s, v),
\end{aligned}
$$

Thus, we see that in order to achieve decay in (1.17) for any $c>0$, one needs to achieve an upper-bound like ${ }^{2}$

$$
F_{1}(-s, v) \leq \exp \left(-\frac{2}{3 \pi} v s^{3 / 2}(1+o(1))\right)
$$

for some choice of $v$. Obtaining (1.18) for optimal $v$ will be a major technical focus of this article. An important step towards this end is Theorem 1.7 below. Before giving this result, we must first uncover a connection to the thinned GOE/GUE point processes with parameter $\gamma:=\gamma(v)=1-e^{-v}$ and the Ablowitz-Segur solution to the Painlevé II equation (this connection is developed further in Section 4).

The Ablowitz-Segur $(\mathrm{AS})$ solution $u_{\mathrm{AS}}(\cdot, \gamma)$ to the Painlevé II equation is a one parameter family of solutions to

with the boundary condition

$$
u_{\mathrm{AS}}^{\prime \prime}=x u_{\mathrm{AS}}+2 u_{\mathrm{AS}}^{3}
$$

$$
u_{\mathrm{AS}}(x ; \gamma)=\sqrt{\gamma} \frac{x^{-1 / 4}}{2 \sqrt{\pi}} e^{-\frac{2}{3} x^{3 / 2}}(1+o(1))
$$

${ }^{2}$ Here, we use "little-Oh" notation: $f(s)$ is called $o(1)$ if $\lim _{s \rightarrow \infty} f(s)=0$. 
as $x \rightarrow \infty$. When $\gamma=1, u_{\mathrm{AS}}$ is called the Hastings-McLeod solution and typically denoted $u_{\mathrm{HM}}$. This particular solution was introduced in [HM80], where they solved the connection problem, that is, gave an asymptotic formula for $u_{\mathrm{HM}}(x)$ as $x \rightarrow-\infty$. For $\gamma \in(0,1)$ fixed, the connection problem for $u_{\mathrm{AS}}$ was partially solved by [AS77a, AS77b].

The thinned version of a point process with parameter $\gamma$ removes each particle independently with probability $1-\gamma$; we discuss the thinned GOE point process formally in Section 4.1. In Theorem 4.4, we prove by way of a Fredholm Pfaffian formula (defined in Section 4.2) that

$$
F_{1}(s, v)=\mathcal{F}_{1}(s, v) \text {, for all } s \in \mathbb{R} \text { and } v \geq 0,
$$

where $\mathcal{F}_{1}(s, v)$ denotes the distribution function of the largest particle of the thinned GOE point process with parameter $\gamma(v)$. Let $\mathcal{F}_{2}(s, v)$ denote the distribution function of the largest particle of the thinned GUE point process with parameter $\gamma(v)$. In Proposition 4.1, we recall a formula from [BB18] that relates $\mathcal{F}_{1}(s, v)$ to $\mathcal{F}_{2}(s, 2 v)$ and $u_{\mathrm{AS}}$, described in the next subsection. It is a result of [CG20], restated here as Proposition 4.2, that

$$
\mathcal{F}_{2}(s, v)=F_{2}(s, v):=\mathbb{E}\left[\exp \left(-v \chi^{\mathrm{Ai}}([s, \infty))\right)\right], \text { for all } s \in \mathbb{R} \text { and } v \geq 0 .
$$

Combining Proposition 4.1, Proposition 4.2, and Theorem 4.4 yields Theorem 1.7, which yields a formula for $F_{1}(s, v)$ in terms of $F_{2}(s, v)$ and $u_{\mathrm{AS}}$. Theorem 1.7 is proved in Section 4.3.

Theorem 1.7. Fix any $s \in \mathbb{R}$ and $v \geq 0$. Define $\gamma:=\gamma(v)=1-e^{-v}$ and $\gamma_{2}:=\gamma_{2}(v)=1-e^{-2 v}$; note that $\gamma_{2} \in[0,1)$. Then

$$
F_{1}(s, v)=\sqrt{F_{2}(s, 2 v)} \sqrt{1+\frac{\cosh \mu\left(s, \gamma_{2}\right)-\sqrt{\gamma_{2}} \sinh \mu\left(s, \gamma_{2}\right)-1}{2-\gamma}}
$$

where

$$
\mu\left(s, \gamma_{2}\right):=\int_{s}^{\infty} u_{\mathrm{AS}}\left(x ; \gamma_{2}\right) d x
$$

In Corollary 5.1, we give an asymptotic expansion for $F_{1}(s, v)$ for any fixed $v>0$ that satisfies (1.18), thus yielding exponential decay on the right-hand of (1.17) with exponent $-s^{3 / 2}$. This yields equation (1.32) of Theorem 1.11. However, the authors of [CG20] found optimum decay of $F_{2}(s, 2 v)$ when $v=\frac{1}{2} s^{\frac{3}{2}-\delta}$. Indeed, part of [CG20, Theorem 1.7] (recorded here as Proposition 4.2) states that, for any $\delta \in(0,2 / 5)$, as $s \rightarrow \infty$,

$$
F_{2}(-s, 2 \bar{v}) \leq \exp \left(-\frac{2}{3 \pi} s^{3-\delta}+\mathcal{O}\left(s^{3-\frac{13 \delta}{11}}\right)\right) .
$$

Fix $\delta \in(0,2 / 5)$. Throughout this paper, we fix

$$
\bar{v}:=\bar{v}(s, \delta)=\frac{1}{2} s^{\frac{3}{2}-\delta} \quad \text { and } \quad \bar{\gamma}:=\gamma_{2}(\bar{v})=1-\exp \left(-s^{\frac{3}{2}-\delta}\right) .
$$

Now, take $v:=\bar{v}$ in the notation of Theorem 1.7. Then upon substituting (1.21) into Theorem 1.7, we see that obtaining the bound

$$
\exp \left(|\mu(-s, \bar{\gamma})|=\exp \left(o\left(s^{3-\delta}\right)\right)\right.
$$

would actually yield (1.18) with $v=\bar{v}$ there. The result would be exponential decay on the righthand side (1.17) with exponent $-s^{3-\delta}$ instead of $-s^{3 / 2}$. Thus, showing (1.23) translates directly into a vastly improved bound on the right-hand of (1.17).

To achieve (1.23), one needs to control $u_{\mathrm{AS}}(x ; \bar{\gamma})$ for all $x \in[-s, \infty)$ and $s \rightarrow \infty$. While much is known about both $u_{\mathrm{AS}}(x ; \gamma)$ and $\mu(s ; \gamma)$ for values of $\gamma$ fixed (with respect to $x$ ), much less is understood for general values of $\gamma$. As we show in the following subsection, there is a particular region of $x$, known as the Stokes region, on which leading-order asymptotics of $u_{\mathrm{AS}}(x ; \bar{\gamma})$ do not exist at this time. This lack of knowledge prevents us from bounding in absolute value the integral 
of $u_{\mathrm{AS}}(x ; \bar{\gamma})$ on the Stokes region, and therefore, we can not establish (1.23); however, we show that given crude leading-order asymptotics on $u_{\mathrm{AS}}$ in the Stokes region (see Conjecture 1), we can obtain (1.23).

1.3. Asymptotics of the Ablowitz-Segur solution to the Painlevé II equation. In this subsection, we recall what is known and unknown about the asymptotic properties of the AblowitzSegur solution to the Painlevé II equation as both $x$ and $\gamma$ vary and detail what these results imply for $F_{1}(s, v)$.

As explained in the last paragraph of the previous subsection, we are interested in $u_{\mathrm{AS}}(x ; \bar{\gamma})$ over $x \in[-s, \infty)$, where $\bar{\gamma}:=1-\exp \left(-s^{\frac{3}{2}-\delta}\right)$, for any $\delta \in(0,2 / 5)$. Our goal is to show (1.23), for which we seek appropriate leading-order asymptotics of $u_{\mathrm{AS}}(x ; \bar{\gamma})$ as $x \rightarrow-\infty$. To understand $u_{\mathrm{AS}}(x ; \gamma)$ for $\gamma$ that may vary with $x$, we turn to the important work of Bothner [Bot17], which contains the most up-to-date results on such asymptotics in the case $x \rightarrow-\infty$ and $|\gamma| \uparrow 1$ (regular transition in [Bot17]) or the case $x \rightarrow-\infty$ and $|\gamma| \downarrow 1$ (singular transition in [Bot17]). These results were achieved via a non-linear steepest descent analysis applied to a certain Riemann-Hilbert problem. Since $s \rightarrow \infty$, we are interested in the regular transition results of [Bot17]. To state these results, we define the following parameter for any $x \in \mathbb{R}$ and $\gamma \in[0,1)$ :

$$
\aleph:=\aleph(x, \gamma)=\frac{-1}{(-x)^{3 / 2}} \log (1-\gamma) .
$$

Note that the exponential decay in (1.19) implies that for any constant $x_{0}>0$, the integral of $u_{\mathrm{AS}}(x ; \bar{\gamma})$ over $\left[-x_{0}, \infty\right)$ is bounded. The remaining region $x \in\left[-s,-x_{0}\right)$ is contained in $\aleph \in(0, \infty)$. For any $\zeta \in\left(0, \frac{2 \sqrt{2}}{3}\right)$, Theorems 1.10 and 1.12 of [Bot17] achieve asymptotic expressions for $u_{\mathrm{AS}}(x ; \gamma)$ as $x \rightarrow-\infty$ in the regions $\aleph \in I_{1}(\zeta):=\left(0, \frac{2 \sqrt{2}}{3}-\zeta\right]$ and $\aleph \in I_{2}:=\left[\frac{2 \sqrt{2}}{3}, \infty\right)$, respectively. ${ }^{3}$ [Bot17, Theorem 1.12] is transcribed here as Proposition 4.6. [Bot17, Theorem 1.10] gives an expression in terms of Jacobi theta functions and elliptic integrals that is pseudoperiodic. In Lemma 4.5, we manipulate this result to show that there exists $\zeta_{0} \in\left(0, \frac{2 \sqrt{2}}{3}\right)$ such that $u_{\mathrm{AS}}(x ; \bar{\gamma})=\mathcal{O}\left((-x)^{1 / 2}\right)$ uniformly over $\aleph \in\left(0, \frac{2 \sqrt{2}}{3}-\zeta_{0}\right]$ as $x \rightarrow-\infty$. From Lemma 4.5 and Proposition 4.6, it follows almost immediately that

$$
\int_{\aleph \in I_{1}\left(\zeta_{0}\right) \cup I_{2}}\left|u_{\mathrm{AS}}(x ; \bar{\gamma})\right| d x=\mathcal{O}\left(s^{3 / 2}\right) .
$$

In [Bot17], $I_{1}(\zeta)$ is named the regular Boutroux region and $I_{2}$ the Hastings-McLeod region; the remaining region of $\aleph>0$ was named the Stokes region.

Definition 1.8 (Stokes region). For any $\zeta \in\left(0, \frac{2 \sqrt{2}}{3}\right)$, the region $\aleph \in\left(\frac{2 \sqrt{2}}{3}-\zeta, \frac{2 \sqrt{2}}{3}\right)$ is referred to as the Stokes region.

[Bot17] does not give a full asymptotic expression for $u_{\mathrm{AS}}(x ; \gamma)$ in the Stokes region, stating that "the nonlinear steepest descent analysis becomes increasingly difficult." Moreover, at the time of this paper's release, it appears that no progress has been made towards such results in the Stokes region [Bot]. As a result, not enough is currently known about $u_{\mathrm{AS}}$ in the Stokes region to obtain (1.23), and thus we can not at present achieve (1.18) with $v=\frac{1}{2} s^{\frac{3}{2}-\delta}$ for any $\delta \in(0,2 / 5)$.

However, observe that only a crude upper-bound on $u_{\mathrm{AS}}(x ; \bar{\gamma})$ is needed in order to show (1.23). Indeed, for $\bar{\aleph}:=\aleph(x, \bar{\gamma})$, the part of the Stokes region that we are interested in is $\left(\frac{2 \sqrt{2}}{3}-\zeta_{0}, \frac{2 \sqrt{2}}{3}\right)$, which is equivalent to

$$
x \in \mathbf{I}_{0}:=\mathbf{I}_{0}(s, \delta)=\left(-\left(\frac{2 \sqrt{2}}{3}-\zeta_{0}\right)^{-2 / 3} s^{1-\frac{2}{3} \delta},-\left(\frac{2 \sqrt{2}}{3}\right)^{-2 / 3} s^{1-\frac{2}{3} \delta}\right) .
$$

${ }^{3}$ Actually, the expression holds for any fixed $f \in \mathbb{R}$ and $I_{2}(f):=\left[\frac{2 \sqrt{2}}{3}-\frac{f}{(-x)^{3 / 2}}, \infty\right)$. However, considering $f$ large (but fixed) does not change our results asymptotically, and so we simply take $f=0$. 
Note that $\mathbf{I}_{0}$ has length $C s^{1-\frac{2}{3} \delta}$, where $C$ denotes some constant.

Conjecture 1. Fix $\delta \in(0,2 / 5)$. Recall $\bar{\gamma}:=\bar{\gamma}(s, \delta)$ from (1.22), and recall $\mathbf{I}_{0}:=\mathbf{I}_{0}(s, \delta)$ from (1.26). As $s \rightarrow \infty$, we have the following uniformly over all $x \in \mathbf{I}_{0}$ (equivalently, $\bar{\aleph}:=$ $\left.\aleph(x, \bar{\gamma}) \in\left(\frac{2 \sqrt{2}}{3}-\zeta_{0}, \frac{2 \sqrt{2}}{3}\right)\right)$ :

$$
\left|u_{\mathrm{AS}}(x ; \bar{\gamma})\right|=o\left(s^{2-\frac{\delta}{3}}\right) .
$$

Assuming Conjecture 1, we immediately have

$$
\int_{\mathbf{I}_{0}}\left|u_{\mathrm{AS}}(x ; \bar{\gamma})\right| d x=o\left(s^{3-\delta}\right),
$$

so that (1.23) follows from (1.25) and the last display. To be precise, we have the following results.

Lemma 1.9. Fix $\delta \in(0,2 / 5)$. Recall the function $\mu$ from Theorem 1.7. There exist positive constants $\mathcal{C}:=\mathcal{C}(\delta)$ and $S_{0}:=S_{0}(\delta)$ such that for all $s \geq S_{0}$,

$$
|\mu(-s, \bar{\gamma})| \leq \mathcal{C} s^{3 / 2}+\left|\int_{\mathbf{I}_{0}} u_{\mathrm{AS}}(x ; \bar{\gamma}) d x\right| .
$$

Assuming Conjecture 1, we have the following expression as $s \rightarrow \infty$,

$$
|\mu(-s, \bar{\gamma})|=o\left(s^{3-\delta}\right) .
$$

Lemma 1.9 is proved in Section 4.4. Combining this result with Theorem 1.7 and (1.21) will yield the following bound.

Theorem 1.10. Assume Conjecture 1. For $\delta \in(0,2 / 5)$, we have the following expression as $s \rightarrow \infty$

$$
F_{1}\left(-s, \frac{1}{2} s^{\frac{3}{2}-\delta}\right) \leq \exp \left(-\frac{1}{3 \pi} s^{3-\delta}(1+o(1))\right) .
$$

Theorem 1.10 is proved in Section 4.3.

Regarding evidence for the validity of Conjecture 1, we note that a leading-order expression for $u_{\mathrm{AS}}(x ; \bar{\gamma})$ was obtained in [Bot17, Theorem 1.13] for the portion of the Stokes region satisfying

$$
\aleph \geq \frac{2 \sqrt{2}}{3}-f_{3} \frac{\log \left((-x)^{3 / 2}\right.}{(-x)^{3 / 2}},
$$

for any $f_{3}<7 / 6$. The expression shows that $u_{\mathrm{AS}}(x ; \bar{\gamma})=\mathcal{O}\left(x^{1 / 2}\right)$ uniformly over this region of $\aleph$, which is consistent with Conjecture 1. We note further that the bound in (1.27) is much looser than both the aforementioned result and the existing leading-order asymptotics given for $u_{\mathrm{AS}}(x ; \bar{\gamma})$ outside of the Stokes region (Proposition 4.6 and Lemma 4.5). Beyond these observations, we do not attempt to provide further justification for Conjecture 1.

1.4. Main results on the GOE Point Process. Theorems 1.11 and 1.12 establish the first bounds on the fluctuations of $\chi^{\mathrm{GOE}}$ below and above its mean, respectively, and may be of independent interest.

Theorem 1.11. Fix any $\eta>0, c>0$, and $\delta \in(0,2 / 5)$. There exists a positive constant $S_{0}:=$ $S_{0}(\eta, c)$ such that for all $s \geq S_{0}$,

$$
\mathbb{P}\left(\chi^{\mathrm{GOE}}[-s, \infty)-\mathbb{E}\left[\chi^{\mathrm{GOE}}([-s, \infty))\right] \leq-c s^{3 / 2}\right) \leq \exp \left(-\eta s^{3 / 2}\right) .
$$

Furthermore, assuming Conjecture 1 , there exist positive constants $S_{0}:=S_{0}(\delta)$ and $K:=K(\delta)$ such that for all $s \geq S_{0}$ and $c>0$,

$$
\mathbb{P}\left(\chi^{\mathrm{GOE}}\left(\mathfrak{B}_{1}(s)\right)-\mathbb{E}\left[\chi^{\mathrm{GOE}}\left(\mathfrak{B}_{1}(s)\right)\right] \leq-c s^{3 / 2}\right) \leq \exp \left(-\frac{1}{2} c s^{3-\delta}(1+o(1))\right),
$$


where $\mathfrak{B}_{1}(s):=[-s, \infty)$.

Theorem 1.11 is proved in Section 5, essentially by combining (1.17), (1.31), and (5.1).

Theorem 1.12. Consider the intervals

$$
\begin{aligned}
& \mathfrak{B}_{1}(\ell):=[-\ell, \infty), \text { and } \\
& \mathfrak{B}_{k}(\ell):=[-k \ell,-(k-1) \ell) \text { for } k \in \mathbb{Z}_{>1} .
\end{aligned}
$$

Fix $c>0$ and $\delta \in(0,2 / 5)$. There exist $L_{0}:=L_{0}(c, \delta)$ and $\mathcal{C}:=\mathcal{C}(c, \delta)>0$ such that, for all $\ell \geq L_{0}$ and for all $k \in \mathbb{Z}_{\geq 1}$, we have

$$
\mathbb{P}\left(\chi^{\mathrm{GOE}}\left(\mathfrak{B}_{k}(\ell)\right)-\mathbb{E}\left[\chi^{\mathrm{GOE}}\left(\mathfrak{B}_{k}(\ell)\right)\right] \geq c \ell^{3 / 2}\right) \leq \exp \left(-\mathcal{C} \ell^{1-\delta}\right) .
$$

Theorem 1.12 is proved in Section 6 .

1.5. Outline. We now give an outline for the remainder of the article. In Section 2, we prove Theorem 1.4 by realizing the left-hand side of the Laplace transform formula (1.10) as an approximate indicator function for $\mathbb{P}\left(\Upsilon_{T}<-s\right)$. This translates our problem into bounding a multiplicative functional of the GOE point process, i.e., the right-hand side of (1.10). These bounds are given by Proposition 2.2.

We next turn to a fine analysis of the GOE point process, which involves estimating the typical locations of the GOE points in large intervals and bounding their deviations from these locations. In Section 3, we define the GOE point process (and Pfaffian point processes in general), and use known results on its correlation functions to prove Theorem 1.6. We then discuss the important connection with the eigenvalues of the stochastic Airy operator (abbreviated SAO). In particular, the result of [RRV11] (Proposition 3.2 below) gives an equivalence in distribution between the GOE points and the negatives of the SAO eigenvalues. Furthermore, [CG20, Proposition 4.5] (Proposition 3.3 below) establishes an upper bound on deviations of the SAO eigenvalues (uniformly over all eigenvalues) from their "typical locations", which are given by the eigenvalues of the Airy operator. The locations of these deterministic eigenvalues are given by a result of [MT59] (Proposition 3.4 below). Combining Proposition 3.2 and Proposition 3.3 yields Theorem 1.5. Thus, we are able to effectively estimate the locations of individual GOE points.

In Section 4, we turn our attention to the cumulant generating function $F_{1}(-s, v)$ for the GOE point process. The importance of this function was established in equation 1.17 of Section 1.2. Via a Fredholm Pfaffian formula for $F_{1}(-s, v)$, we prove in Theorem 4.4 a key equality between $F_{1}(-s, v)$ and the distribution function of the largest eigenvalue of the thinned GOE point process. This allows us to translate the work of [BB18] on this distribution function to $F_{1}(-s, v)$, which in particular leads to the proofs of Theorem 1.7 and (assuming Lemma 1.9) Theorem 1.10 in Section 4.3. Lemma 1.9 is proved in Section 4.4.

In Sections 5 and 6, we prove Theorems 1.11 and 1.12 respectively. Theorem 1.11 is proved essentially by substituting the results of Corollary 5.1 and Theorem 1.10 into (1.17). Our strategy for proving Theorem 1.12 involves approximating the number of GOE points in a closed interval of length $s$ by carefully estimating the nearest GOE points to the endpoints of this interval, and then bounding the fluctuations of these GOE points via Theorem 1.5.

In Section 7, we apply our work on the GOE point process to prove Proposition 2.2.

Acknowledgements. We are grateful to Ivan Corwin for suggesting this problem and for providing helpful comments on numerous drafts of this paper, to Promit Ghosal for providing guidance and insight at several stages of this project, and to Thomas Bothner for enlightening discussions about the current literature on leading-order asymptotics for the Ablowitz-Segur solution to the Painlevé II equation in various regimes. We are also grateful to Guillaume Barraquand, Pierre le Doussal, Alexandre Krajenbrink, Yier Lin, Baruch Meerson, Li-Cheng Tsai, and Shalin Parekh 
for discussions and conversations related to this work. Finally, we are grateful to the anonymous referees for their time and effort in providing many important comments and suggestions. The author was partially funded by Ivan Corwin's Packard Fellowship for Science and Engineering while working on this paper. The author is currently supported by the National Science Foundation Graduate Research Fellowship under Grant No. 1839302.

\section{Proof of the MAIN THEOREM}

We begin by establishing upper and lower bounds on the right-hand side of the Laplace transform formula (1.10) in Proposition 2.1.

Proposition 2.1. Fix any $\eta>0, \varepsilon \in(0,1 / 3), \delta \in(0,1 / 4)$, and $T_{0}>0$. There exist positive constants $S_{0}:=S_{0}\left(\eta, \varepsilon, \delta, T_{0}\right), C:=C\left(T_{0}\right), K_{1}:=K_{1}\left(\varepsilon, \delta, T_{0}\right)>0$, and $K_{2}:=K_{2}\left(T_{0}\right)>0$ such that for all $s \geq S_{0}$ and $T \geq T_{0}$, we have

$$
\mathbb{E}\left[\exp \left(-\frac{1}{4} \exp \left(T^{1 / 3}\left(\Upsilon_{T}+s\right)\right)\right)\right] \geq e^{-\frac{2(1+C \varepsilon)}{15 \pi} T^{1 / 3} s^{5 / 2}}+e^{-K_{2} s^{3}}
$$

and

$$
\mathbb{E}\left[\exp \left(-\frac{1}{4} \exp \left(T^{1 / 3}\left(\Upsilon_{T}+s\right)\right)\right)\right] \leq e^{-\frac{2(1-C \varepsilon)}{15 \pi} T^{1 / 3} s^{5 / 2}}+e^{-\frac{\varepsilon}{2} s T^{1 / 3}-\eta s^{3 / 2}}+e^{-\frac{1-C \varepsilon}{24} s^{3}} .
$$

Assuming Conjecture 1, we have the stronger upper bound

$$
\mathbb{E}\left[\exp \left(-\frac{1}{4} \exp \left(T^{1 / 3}\left(\Upsilon_{T}+s\right)\right)\right)\right] \leq e^{-\frac{2(1-C \varepsilon)}{15 \pi} T^{1 / 3} s^{5 / 2}}+e^{-\frac{\varepsilon}{2} s T^{1 / 3}-K_{1} s^{3-\delta}}+e^{-\frac{1-C \varepsilon}{24} s^{3}} .
$$

We prove Proposition 2.1 in Section 2.2. We now prove the main result.

2.1. Proof of Theorem 1.4. From Markov's inequality, we have

$$
\begin{aligned}
\mathbb{P}\left(\Upsilon_{T} \leq-s\right) & =\mathbb{P}\left(\exp \left(-\frac{1}{4} \exp \left(T^{1 / 3}\left(\Upsilon_{T}+s\right)\right)\right) \geq e^{-1 / 4}\right) \\
& \leq e^{1 / 4} \mathbb{E}\left[\exp \left(-\frac{1}{4} \exp \left(T^{1 / 3}\left(\Upsilon_{T}+s\right)\right)\right)\right]
\end{aligned}
$$

From the above, we see that (2.2) and (2.3) imply (1.12) and (1.13) of Theorem 1.4, respectively.

We now show that $(2.1)$ yields $(1.11)$. Let $\bar{s}:=(1-\varepsilon)^{-1} s$. Observe that

$$
\begin{aligned}
\Re & :=\mathbb{E}\left[\exp \left(-\frac{1}{4} \exp \left(T^{1 / 3}\left(\Upsilon_{T}+\bar{s}\right)\right)\right)\right] \\
& \leq \mathbb{E}\left[\mathbb{1}\left(\Upsilon_{T} \leq-s\right)+\mathbb{1}\left(\Upsilon_{T}>-s\right) \exp \left(-\frac{1}{4} \exp \left(T^{1 / 3}\left(\Upsilon_{T}+\bar{s}\right)\right)\right)\right] \\
& \leq \mathbb{E}\left[\mathbb{1}\left(\Upsilon_{T} \leq-s\right)+\mathbb{1}\left(\Upsilon_{T}>-s\right) \exp \left(-\frac{1}{4} \exp \left(\varepsilon \bar{s} T^{1 / 3}\right)\right)\right] .
\end{aligned}
$$

The second inequality follows from the fact that $\Upsilon_{T}>-s$ implies $\Upsilon_{T}+\bar{s}>\varepsilon \bar{s}$. Continuing from (2.4), we compute

$$
\Re \leq \mathbb{P}\left(\Upsilon_{T} \leq-s\right)+\exp \left(-\frac{1}{4} \exp \left(\varepsilon \bar{s} T^{1 / 3}\right)\right)
$$

It follows from (2.1) that for all $s \geq S:=S\left(\varepsilon, \delta, T_{0}\right)$ and $T \geq T_{0}$,

$$
\mathfrak{R} \geq \exp \left(-\left(1+C \varepsilon+C^{\prime} \varepsilon\right) \frac{2}{15 \pi} T^{1 / 3} s^{5 / 2}\right)+\exp \left(-K_{2} s^{3}\right) .
$$


Here, the $C^{\prime} \varepsilon$ term appears because $\bar{s}^{5 / 2} \leq s^{5 / 2}\left(1+C^{\prime} \varepsilon\right)$ for some constant $C^{\prime}>0$. We now note that there exists a constant $S^{\prime}:=S^{\prime}\left(\varepsilon, \delta, T_{0}\right)$ such that for all $s \geq S^{\prime}$ and $T \geq T_{0}$,

$$
\begin{aligned}
& \exp \left(\varepsilon \bar{s} T^{1 / 3}\right) \geq T^{1 / 3} \frac{2 s^{5 / 2}}{15 \pi}-\log \varepsilon, \text { and thus } \\
& \exp \left(-\exp \left(\varepsilon \bar{s} T^{1 / 3}\right)\right) \leq \varepsilon \exp \left(-\frac{2}{15 \pi} T^{1 / 3} s^{5 / 2}\right) .
\end{aligned}
$$

Solving for $\mathbb{P}\left(\Upsilon_{T} \leq-s\right)$ in (2.5) and substituting the lower bound (2.6) on $\mathfrak{R}$ and the upper bound (2.7) on $\exp \left(-\exp \left(\varepsilon \bar{s} T^{1 / 3}\right)\right)$ yields, for all $s \geq \max \left\{S, S^{\prime}\right\}$ and for all $T \geq T_{0}$,

$$
\mathbb{P}\left(\Upsilon_{T} \leq-s\right) \geq(1-\varepsilon) \exp \left(-\left(1+\left(C+C^{\prime}\right) \varepsilon\right) \frac{2}{15 \pi} T^{1 / 3} s^{5 / 2}\right)+\exp \left(-K_{2} s^{3}\right) .
$$

The multiplicative factor $(1-\varepsilon)$ can be absorbed into the $\left(1+\left(C+C^{\prime}\right) \varepsilon\right)$ factor on the right-hand side above. Finally, taking $C:=C+C^{\prime}$ yields the right-hand side of (1.11), thus completing the proof of Theorem 1.4.

2.2. Proof of Proposition 2.1. As above, let $\left(a_{1}>a_{2}>\ldots\right)$ denote the GOE point process. Define

$$
\begin{aligned}
I_{s}(x) & :=\frac{1}{\sqrt{1+\exp \left(T^{1 / 3}(x+s)\right)}}, \text { and } \\
J_{s}(x) & :=-\log \left(I_{s}(x)\right)=\frac{1}{2} \log \left(1+\exp \left(T^{1 / 3}(x+s)\right)\right) .
\end{aligned}
$$

We now give upper and lower bounds on $\mathbb{E}_{\mathrm{GOE}}\left[\prod_{k=1}^{\infty} I_{s}\left(\mathrm{a}_{k}\right)\right]$. These bounds and Proposition 1.3 allow us to complete the proof of Proposition 2.1.

Proposition 2.2. Fix any $\eta>0, \varepsilon \in(0,1 / 3), \delta \in(0,1 / 4)$, and $T_{0}>0$. There exist positive constants $S_{0}:=S_{0}\left(\eta, \varepsilon, \delta, T_{0}\right), C:=C\left(T_{0}\right), K_{1}:=K_{1}\left(\varepsilon, \delta, T_{0}\right)>0$, and $K_{2}:=K_{2}\left(T_{0}\right)>0$ such that for all $s \geq S_{0}$ and $T \geq T_{0}$, we have

$$
\mathbb{E}_{\mathrm{GOE}}\left[\prod_{k=1}^{\infty} I_{s}\left(\mathrm{a}_{k}\right)\right] \leq e^{-\frac{2(1-C \varepsilon)}{15 \pi} T^{1 / 3} s^{5 / 2}}+e^{-\frac{\varepsilon}{2} s T^{1 / 3}-\eta s^{3 / 2}}+e^{-\frac{1-C \varepsilon}{24} s^{3}}
$$

and

$$
\mathbb{E}_{\mathrm{GOE}}\left[\prod_{k=1}^{\infty} I_{s}\left(\mathrm{a}_{k}\right)\right] \geq e^{-\frac{2(1+C \varepsilon)}{15 \pi} T^{1 / 3} s^{5 / 2}}+e^{-K_{2} s^{3}} .
$$

Assuming Conjecture 1, we have the stronger upper bound

$$
\mathbb{E}_{\mathrm{GOE}}\left[\prod_{k=1}^{\infty} I_{s}\left(\mathrm{a}_{k}\right)\right] \leq e^{-\frac{2(1-C \varepsilon)}{15 \pi} T^{1 / 3} s^{5 / 2}}+e^{-\frac{\varepsilon}{2} s T^{1 / 3}-K_{1} s^{3-\delta}}+e^{-\frac{1-C \varepsilon}{24} s^{3}}
$$

We complete the proof of (2.11) and (2.12) in Section 7.1, and the proof of(2.10) in Section 7.2. Proof of Proposition 2.1. This follows immediately from Proposition 1.3 and Proposition 2.2.

\section{The GOE POINT PROCESS}

Proposition 2.2 reduces our problem to a question about the GOE point process. In this section, we formally define this process and examine results pertaining to the statistics of the process, such as the distribution of the GOE points, the typical locations of individual points, and deviations away from these typical locations. The results developed here connect the GOE point process to the stochastic Airy operator (see Section 3.2) and will be crucial to the proofs that follow. 
3.1. First notions. The GOE point process, denoted by $\left(\mathrm{a}_{1}>\mathrm{a}_{2}>\ldots\right)$ or $\chi^{\mathrm{GOE}}$, is a simple Pfaffian point process on $(\mathbb{R}, \mathcal{B}(\mathbb{R}), \mu)$, where here $\mu$ denotes Lebesgue measure. We define this object now. We first define point processes via random point configurations (see, for instance, [AGZ10, Section 4.2.1]). Give $\mathbb{R}$ the Borel sigma algebra $\mathcal{B}(\mathbb{R})$ equipped with a positive Radon measure $\mu$ (not necessarily Lebesgue). Let $\operatorname{Conf}(\mathbb{R})$ denote the space of configurations of $\mathbb{R}$, that is, discrete subsets. For any $B \in \mathcal{B}(\mathbb{R})$ and $X \in \operatorname{Conf}(\mathbb{R})$, let $N_{B}(X):=\#\{B \cap X\}$. Endow $\operatorname{Conf}(\mathbb{R})$ with the sigma algebra $\Sigma$ generated by the cylinder sets $C_{n}^{B}:=\left\{X \in \operatorname{Conf}(\mathbb{R}): N_{B}(X)=n\right\}$, for $n \in \mathbb{Z}^{+}$. A point process is a probability measure $\nu$ on $(\operatorname{Conf}(\mathbb{R}), \Sigma)$. [AGZ10, Lemma 4.2.2] shows that a random configuration $X$ with distribution $\nu$ can be associated to a non-negative integer-valued random measure $\chi$ on $(\mathbb{R}, \mathcal{B}(\mathbb{R}), \mu)$ such that

$$
\chi(B)=N_{B}(X),
$$

and this random measure $\chi$ will also be referred to as the point process when clear. A point process is called simple if $\mu(e \in \mathbb{R}: \chi(\{e\})>1)=0$. Intuitively, a simple point process $\chi$ evaluated on a Borel set $B$ counts the number of points contained in $B$ of the designated random configuration.

Now, for $k \geq 1$, consider the measure $\mu_{k}$ on $\mathbb{R}^{k}$ such that for disjoint Borel sets $B_{1}, \ldots, B_{k} \in \mathcal{B}(\mathbb{R})$,

$$
\mu_{k}\left(B_{1} \times \cdots \times B_{k}\right)=\mathbb{E}_{\nu}\left[\#\left\{k \text {-tuples of distinct points } x_{1} \in X \cap B_{1}, \ldots, x_{k} \in X \cap B_{k}\right\}\right] .
$$

Assuming that $\mu_{k}$ is absolutely continuous with respect to $\mu^{\otimes k}$, we define the $k$-point correlation function $\rho_{k}$ of $\chi$ to be the Radon-Nykodym derivative of $\mu_{k}$ with respect to $\mu^{\otimes k}$. This is a locally integrable function $\rho_{k}: \mathbb{R}^{k} \rightarrow[0, \infty)$ such that, for measurable functions $f: \mathbb{R} \rightarrow \mathbb{C}$, we have

$$
\mathbb{E}_{\nu}\left[\sum_{\left(x_{1}, \ldots, x_{k}\right) \in X^{k}} f\left(x_{1}\right) \ldots f\left(x_{k}\right)\right]=\int_{\mathbb{R}^{k}} \rho_{k}\left(x_{1}, \ldots, x_{k}\right) f\left(x_{1}\right) \ldots f\left(x_{k}\right) \mathrm{d} \mu^{\otimes k} .
$$

Here, $X$ is a random configuration with law $\nu$. One might note that our definition of $\rho_{k}$ does not specify its value on points $\left(x_{1}, \ldots, x_{k}\right)$ where $x_{i}=x_{j}$ for some $i \neq j$. On such points, we set $\rho_{k}=0$; to understand the reasoning behind this, see [AGZ10, Remark 4.2.4]. We call $\chi$ a Pfaffian point process if there exists a $2 \times 2$ skew-symmetric matrix-kernel $K: \mathbb{R}^{2} \rightarrow M_{2}(\mathbb{C})$ such that

$$
\rho_{k}\left(x_{1}, \ldots, x_{k}\right)=\operatorname{Pf}\left[K\left(x_{i}, x_{j}\right)\right]_{i, j=1}^{k},
$$

where Pf denotes the Pfaffian.

The GOE point process is the simple Pfaffian point process with correlation kernel $K^{\mathrm{GOE}}$, whose explicit form can be found, for instance, in [BBCW18, Definition 6.1] (we will not need the explicit form of $K^{\mathrm{GOE}}$ here). The GOE point process can be constructed as the limiting point process of the largest eigenvalues of the GOE $n \times n$ ensemble under so-called edge scaling, that is, centering by $2 \sqrt{n}$ and scaling by $n^{1 / 6}$. We write $\chi^{\mathrm{GOE}}$ to denote the associated random measure and $\rho_{k}^{\mathrm{GOE}}$ to denote the $k^{\text {th }}$ correlation function of the GOE point process. We also write $\left(\mathrm{a}_{1}>\mathrm{a}_{2}>\ldots\right)$ to denote the random configuration of GOE points.

Proposition 1.3 and the work achieved in Section 2.1 show that studying the GOE point process can serve as a proxy for studying the lower tail of the half-space KPZ equation. Theorem 1.6 establishes a basic statistic of the GOE point process: its expectation on the interval $[-s, \infty)$, for any $s>0$. We now prove this theorem.

Proof of Theorem 1.6. Note that for any point process $\chi$ with one-point correlation function $\rho_{1}$, we have on any interval $I \subseteq \mathbb{R}$,

$$
\mathbb{E}[\chi(I)]=\int_{I} \rho_{1}(x) d x
$$


Thus, we have

$$
\mathbb{E}_{\mathrm{GOE}}\left[\chi^{\mathrm{GOE}}\left(\mathfrak{B}_{1}(s)\right)\right]=\int_{-s}^{\infty} \rho_{1}^{\mathrm{GOE}}(x) d x,
$$

for $s>0$. Let $\rho_{1}^{\mathrm{GUE}}$ denote the one-point correlation function for $\chi^{\mathrm{GUE}}$. From Equations (7.67) and (7.147) of [For10], we have the relation ${ }^{4}$

$$
\rho_{1}^{\mathrm{GOE}}(x)=\rho_{1}^{\mathrm{GUE}}(x)+\frac{1}{2} \operatorname{Ai}(x)\left(1-\int_{x}^{\infty} \operatorname{Ai}(t) d t\right),
$$

where $\operatorname{Ai}(\cdot)$ denotes the Airy function. Since $\int_{-\infty}^{\infty} \operatorname{Ai}(t) d t=1$ ([Nis, Equation 9.10.11]), we may write $(3.3)$ as

$$
\rho_{1}^{\mathrm{GOE}}(x)=\rho_{1}^{\mathrm{GUE}}(x)+\frac{1}{2} \operatorname{Ai}(x) \int_{-\infty}^{x} \operatorname{Ai}(t) d t .
$$

Now, [For10, Equation 7.69], [Nis, Equation 9.7.9], and [Nis, Equation 9.10.6] yield the following asymptotic expansions for $\rho_{1}^{\mathrm{GUE}}(x), \operatorname{Ai}(x)$, and $\int_{-\infty}^{x} \operatorname{Ai}(t) d t$ respectively, as $x \rightarrow-\infty$ :

$$
\begin{aligned}
\rho_{1}^{\mathrm{GUE}}(x) & =\frac{\sqrt{-x}}{\pi}-\frac{\cos \left(\frac{4}{3}(-x)^{3 / 2}\right)}{4 \pi(-x)}+\mathcal{O}\left((-x)^{-5 / 2}\right), \\
\operatorname{Ai}(x) & =\frac{\cos \left(\frac{2}{3}(-x)^{3 / 2}-\frac{\pi}{4}\right)}{\sqrt{\pi}(-x)^{1 / 4}}+\mathcal{O}\left((-x)^{-7 / 4}\right), \text { and } \\
\int_{-\infty}^{x} \operatorname{Ai}(t) d t & =\frac{\cos \left(\frac{2}{3}(-x)^{3 / 2}+\frac{\pi}{4}\right)}{\sqrt{\pi}(-x)^{3 / 4}}+\mathcal{O}\left((-x)^{-9 / 4}\right) .
\end{aligned}
$$

Substituting (3.5)-(3.7) into (3.4) yields

$$
\rho_{1}^{\mathrm{GOE}}(x)=\frac{\sqrt{-x}}{\pi}+\mathcal{O}\left((-x)^{-5 / 2}\right)
$$

as $x \rightarrow-\infty$ (note that the cosine terms above cancel with one another after substitution into (3.4)). It follows that

$$
\int_{-s}^{-1} \rho_{1}^{\mathrm{GOE}}(x) d x=\frac{2}{3 \pi} s^{3 / 2}+\mathfrak{D}_{1}(s)
$$

where $\mathfrak{D}_{1}(s)$ satisfies $\sup _{s>0}|\mathfrak{D}(s)|<\infty$.

Next, because $\rho_{1}^{\mathrm{GUE}}(x), \operatorname{Ai}(x)$, and $\int_{-\infty}^{x} \operatorname{Ai}(t) d t$ are bounded over $x \in[-1,0]$, we have

$$
\int_{-1}^{0} \rho_{1}^{\mathrm{GOE}}(x) d x=\mathfrak{D}_{2}
$$

for some constant $\mathfrak{D}_{2}<\infty$.

It remains to handle the integral of $\rho_{1}^{\mathrm{GOE}}(x)$ over $x \in[0, \infty)$. [For10, Equation 7.72] states that

$$
\rho_{1}(x)=e^{-4 x^{3 / 2} / 3}(1+o(1)),
$$

and thus we have $\int_{0}^{\infty} \rho_{1}^{\mathrm{GUE}}(x) d x=\mathfrak{D}_{3}$, for some constant $\mathfrak{D}_{3}$. Recall that $\operatorname{Ai}(x) \geq 0$ for $x \geq 0$. It then follows from (3.4) and the triangle inequality that

$$
\left|\int_{0}^{\infty} \rho_{1}^{\mathrm{GOE}}(x) d x\right| \leq\left|\mathfrak{D}_{3}\right|+\frac{1}{2} \int_{0}^{\infty} \operatorname{Ai}(x)\left|\int_{-\infty}^{x} \operatorname{Ai}(t) d t\right| d x
$$

\footnotetext{
$\overline{4}\left[\right.$ For10, Equation 7.147] writes this equation with " $K^{\text {soft }}(x, x)$ " instead of $\rho_{1}^{\text {GUE }}(x)$, as we have here, where $K^{\text {soft }}(\cdot, \cdot)$ is defined in [For10, Equation 7.12]. Our expression follows from [For10, Equation 7.67], which shows that $K^{\text {soft }}(x, x)=$ $\rho_{1}^{\mathrm{GUE}}(x)$, for any $x \in \mathbb{R}$.
} 
Since $\int_{-\infty}^{\infty} \operatorname{Ai}(t) d t=1, \int_{-\infty}^{0} \operatorname{Ai}(t) d t=2 / 3$ ([Nis, Equation 9.10.11]), and $\operatorname{Ai}(t) \geq 0$ for $t \geq 0$, we have $\left|\int_{-\infty}^{x} \operatorname{Ai}(t) d t\right| \leq\left|\int_{-\infty}^{\infty} \operatorname{Ai}(t) d t\right|=1$ for all $x \geq 0$. It then follows from (3.10) and the identity $\int_{0}^{\infty} \operatorname{Ai}(t) d t=1 / 3$ that

$$
\int_{0}^{\infty} \rho_{1}^{\mathrm{GOE}}(x) d x=\mathfrak{D}_{4}
$$

for some constant $\mathfrak{D}_{4}$. Combining equations (3.2), (3.8), (3.9), and (3.11) yields

$$
\mathbb{E}_{\mathrm{GOE}}\left[\chi^{\mathrm{GOE}}\left(\mathfrak{B}_{1}(s)\right)\right]=\frac{2}{3 \pi} s^{3 / 2}+D_{1}(s),
$$

where $D_{1}(s)=\mathfrak{D}_{1}(s)+\mathfrak{D}_{2}+\mathfrak{D}_{4}$, and therefore clearly satisfies $\sup _{s>0}|D(s)|<\infty$. Thus, we have (1.16).

3.2. The $\beta$ stochastic Airy operator. We now apply and enhance the tools developed in [CG20, Section 4.3] to connect the GOE point process with the eigenvalues of the stochastic Airy operator $\mathcal{H}_{\beta}$ with $\beta=1$. Observed in [ES07] and proved in [RRV11, Theorem 1.1], Proposition 3.2 gives an equivalence in distribution between the eigenvalues of $\mathcal{H}_{\beta}$ and the negatives of the GOE points. Proposition 3.3 below was proved in [CG20, Proposition 4.5], and establishes a uniform bound on the deviations of the (random) $\mathcal{H}_{\beta}$ eigenvalues from the eigenvalues of the (deterministic) Airy operator, and Theorem 1.5 establishes the same uniform bound on deviations of the GOE points from these deterministic eigenvalues. Finally, Proposition 3.4, which was proved in [MT59], approximates the location of each eigenvalue of the Airy operator.

We now define the stochastic Airy operator through the theory of Schwartz distributions.

Definition 3.1 (stochastic Airy operator). Let $D:=D\left(\mathbb{R}^{+}\right)$denote the space of distributions, i.e., the continuous dual of the space of smooth, compactly supported test functions equipped with the topology of uniform convergence of all derivatives on compact sets. All formal derivatives of any continuous function $f$ are distributions, with action on any test function $\phi \in C_{0}^{\infty}$ given by integration by parts as follows:

$$
\prec \phi, f^{(k)}(x) \succ:=(-1)^{k} \int f(x) \phi^{(k)}(x) d x,
$$

where $\prec \cdot, \cdot \succ$ is notation not to be confused with the $L^{2}$ inner product $\langle\cdot, \cdot\rangle$. In particular, since Brownian motion $B$ is a random continuous function, its formal derivative $B^{\prime}$ is a random element of $D$. The $\beta>0$ stochastic Airy operator is a random linear map

$$
\mathcal{H}_{\beta}: H_{\mathrm{loc}}^{1} \rightarrow D
$$

such that

$$
\mathcal{H}_{\beta} f=-f^{(2)}+x f+\frac{2}{\sqrt{\beta}} f B^{\prime}
$$

where $H_{\text {loc }}^{1}$ is the space of functions $f: \mathbb{R}^{+} \rightarrow \mathbb{R}$ such that for any compact $I, f^{\prime} \mathbb{1}(I) \in L^{2}$. Though $D$ is only closed under multiplication by smooth functions and $f \in H_{\mathrm{loc}}^{1}$, we make sense of $f B^{\prime}$ as the derivative of $\int_{0}^{y} f B^{\prime} d x:=-\int_{0}^{y} B f^{\prime} d x+f(y) B_{y}-f(0) B_{0}$. The Airy operator $\mathcal{A}:=-\partial_{x}^{2}+x$ is the non-random part of $\mathcal{H}_{\beta}$.

To define the eigenvalues/eigenfunctions of $\mathcal{H}_{\beta}$, we define the Hilbert space $L^{*}$ with norm

$$
\|f\|_{*}^{2}=\int_{0}^{\infty}\left(\left(f^{\prime}\right)^{2}+(1+x) f^{2}\right) d x, \quad L^{*}:=\left\{f: f(0)=0,\|f\|_{*}<\infty\right\} .
$$

We say a pair $(f, \Lambda) \in L^{*} \times \mathbb{R}$ is an eigenfunction/eigenvalue pair for $\mathcal{H}_{\beta}$ if $\mathcal{H}_{\beta} f=\Lambda f$. 
The following is a special case of [RRV11, Theorem 1.1], namely, the $\beta=1$ case. $^{5}$

Proposition 3.2 ([RRV11, Theorem 1.1]). Let $\left(\Lambda_{1}<\Lambda_{2}<\ldots\right)$ denote the eigenvalues of $\mathcal{H}_{1}$, and recall that $\left(\mathrm{a}_{1}>\mathrm{a}_{2}>\ldots\right)$ denotes the GOE point process. Then for any $k \geq 1$, we have

$$
\left(-\mathrm{a}_{1}, \ldots,-\mathrm{a}_{k}\right) \stackrel{(\mathrm{d})}{=}\left(\Lambda_{1}, \ldots, \Lambda_{k}\right) .
$$

[RRV11] and [Vir14] show that there exists a random band with uniform width $C_{\varepsilon}$ around each eigenvalue of the Airy operator such that each eigenvalue of $\mathcal{H}_{\beta}$ is contained in the band around the corresponding Airy operator eigenvalue.

Proposition 3.3 ([CG20, Proposition 4.5]). Denote the eigenvalues of the Airy operator $\mathcal{A}$ by $\left(\lambda_{1}<\lambda_{2}<\ldots\right)$ and the eigenvalues of $\mathcal{H}_{\beta}$ by $\left(\Lambda_{1}^{\beta}<\Lambda_{2}^{\beta}<\ldots\right)$. For any $\varepsilon \in(0,1)$, define the random variable $C_{\varepsilon}$ as the smallest real number such that for all $k \geq 1$,

$$
(1-\varepsilon) \lambda_{k}-C_{\varepsilon} \leq \Lambda_{k}^{\beta} \leq(1+\varepsilon) \lambda_{k}+C_{\varepsilon} .
$$

Then for all $\varepsilon, \delta \in(0,1)$, there exist positive constants $S_{0}:=S_{0}(\varepsilon, \delta)$, and $\kappa:=\kappa(\varepsilon, \delta)$ such that for all $s \geq S_{0}$,

$$
\mathbb{P}\left(C_{\varepsilon} \geq \frac{s}{\sqrt{\beta}}\right) \leq \kappa \exp \left(-\kappa s^{1-\delta}\right)
$$

Proposition 3.3 gives an exponential upper-tail bound on $C_{\varepsilon}$ that will be crucial to our proof of Theorem 1.12. Note that Theorem 1.5 follows immediately from Propositions 3.2 and 3.3.

To prove Theorem 1.12, we will also need the following results on the approximate location of eigenvalues of the Airy operator $\mathcal{A}=-\partial_{x}^{2}+x$.

Proposition 3.4 ([MT59]). If the eigenvalues of the Airy operator $\mathcal{A}$ are denoted by $\left(\lambda_{1}<\lambda_{2}<\right.$ $\ldots)$, then for all $n \geq 1$, we have

$$
\lambda_{n}=\left(\frac{3 \pi}{2}\left(n-\frac{1}{4}+\mathcal{R}(n)\right)\right)^{2 / 3},
$$

where for some large constant $K \in \mathbb{R}$, we have

$$
|\mathcal{R}(n)| \leq K / n
$$

Corollary 3.5. For any $T \in \mathbb{R}_{\geq 0}$, define $k:=k(T)=\#\left\{n: \lambda_{n} \leq T\right\}$. We have

$$
k=\frac{2}{3 \pi} T^{3 / 2}+C_{1}(T),
$$

where $\sup _{x>0}\left|C_{1}(x)\right|<1$; thus,

$$
k-\mathbb{E}\left[\chi^{\mathrm{GOE}}[-T, \infty)\right]=\mathcal{O}_{T}(1) .
$$

Proof. From (3.15), it is clear that $k=\lfloor x\rfloor$, where $x \in \mathbb{R}_{\geq 0}$ satisfies

$$
T=\left(\frac{3 \pi}{2}\left(x-\frac{1}{4}+\mathcal{R}(x)\right)\right)^{2 / 3} \text {. }
$$

Solving for $x$ gives

$$
x=\frac{2}{3 \pi} T^{3 / 2}+\frac{1}{4}+\mathcal{R}(x) .
$$

Recall from Proposition 3.4 that $|\mathcal{R}(x)| \leq K / x$. As $T$ approaches $\infty$, we have $x \sim \frac{2}{3 \pi} T^{3 / 2}$, and thus $k$ will simply be the the closest integer to $\frac{2}{3 \pi} T^{3 / 2}+\frac{1}{4}$. From the expression $\mathbb{E}\left[\chi^{\mathrm{GOE}}[-T, \infty)\right]=$ $\frac{2}{3 \pi} T^{3 / 2}+D_{1}(T)$ given by Theorem 1.6, the corollary follows.

${ }^{5}$ The result is proved for any $\beta$ : under edge scaling, the $k$ largest eigenvalues of the $n \times n$ Hermite $\beta$-ensemble converge jointly in distribution to the smallest $k$ eigenvalues of $\mathcal{H}_{\beta}$ as $n \rightarrow \infty$. 


\section{The Cumulant generating FunCtion for $\chi^{\mathrm{GOE}}$}

The proof of Theorem 1.11, which makes up the contents of Section 5, will boil down to estimating the cumulant generating function for $\chi^{\mathrm{GOE}}$,

$$
F_{1}(s, v):=\mathbb{E}\left[\exp \left(-v \chi^{\mathrm{GOE}}([s, \infty))\right)\right] .
$$

The main result of this section is Theorem 4.4, which connects $F_{1}(s, v)$ to the distribution function of the largest eigenvalue of the thinned GOE point process via a Fredholm Pfaffian. Theorem 4.4 is a major input towards Corollary 5.1 and Theorem 1.10, which provide the needed bounds on $F_{1}(s, v)$ to prove Theorem 1.11 in Section 5 .

4.1. The thinned GOE point process and the Painlevé II equation. Theorem 4.4 equates $F_{1}(s, v)$ to the distribution function $\mathcal{F}_{1}(s, v)$ of the largest particle $\mathrm{a}_{1}(\gamma)$ of the thinned GOE point process with parameter $\gamma:=1-e^{-v}$. This is the point process obtained by independently removing each particle of the GOE point process (see Section 3) with probability $1-\gamma$. We may similarly define the thinned GUE point process and the distribution function $\mathcal{F}_{2}(s, v)$ of the largest particle of the thinned GUE point process with parameter $\gamma$. Note that, like the GOE point process, the thinned GOE point process is simple and Pfaffian. To see that it is Pfaffian, let $\left\{Y_{i}\right\}_{i \in \mathbb{N}}$ be a sequence of i.i.d. Bernoulli random variables such that $\mathbb{P}\left(Y_{1}=1\right)=\gamma$. Let $\nu^{\mathrm{GOE}}$ and $\nu^{\text {thin }}$ be the laws on $\operatorname{Conf}(\mathbb{R})$ associated to the GOE and thinned GOE point processes respectively, and let $X$ and $\hat{X}$ be random configurations with laws $\nu^{\mathrm{GOE}}$ and $\nu^{\text {thin }}$ respectively. Then, for a measurable function $f: \mathbb{R} \rightarrow \mathbb{C}$, we have

$$
\mathbb{E}\left[\sum_{\left(x_{1}, \ldots, x_{k}\right) \in \hat{X}^{k}} f\left(x_{1}\right) \ldots f\left(x_{k}\right)\right]=\mathbb{E}\left[\sum_{\left(x_{1}, \ldots, x_{k}\right) \in X^{k}} \prod_{i=1}^{k} f\left(x_{i}\right) Y_{i}\right]=\gamma^{k} \mathbb{E}\left[\sum_{\left(x_{1}, \ldots, x_{k}\right) \in X^{k}} \prod_{i=1}^{k} f\left(x_{i}\right)\right],
$$

where the last equality follows from the independence of the $Y_{i}$ from each other and from the GOE point process. We then have from (3.1) that, for any $k \geq 1$,

$$
\rho_{k}^{\text {thin }}=\gamma^{k} \rho_{k}^{\mathrm{GOE}},
$$

where $\rho_{k}^{\text {thin }}$ denotes the $k^{\text {th }}$ correlation functions for the thinned GOE point process. Furthermore, it follows that the correlation kernel for the thinned GOE point process is $\gamma K^{\mathrm{GOE}}$.

Proposition 4.1 below gives a formula for $\mathcal{F}_{1}(s, v)$ in terms of $\mathcal{F}_{2}(s, v)$ and a certain integral of the Ablowitz-Segur (AS) solution $u_{\mathrm{AS}}(\cdot, \gamma)$ to the Painlevé II equation. Recall from Section 1.3 that $u_{\mathrm{AS}}$ is a one-parameter family of solutions to

$$
u_{\mathrm{AS}}(s, \gamma)^{\prime \prime}=x u_{\mathrm{AS}}(s, \gamma)+2 u_{\mathrm{AS}}^{3}(s, \gamma)
$$

with boundary coundition

$$
u_{\mathrm{AS}}(s, \gamma)=\sqrt{\gamma} \frac{s^{-1 / 4}}{2 \sqrt{\pi}} e^{-\frac{2}{3} s^{3 / 2}}(1+o(1)), \text { as } s \rightarrow \infty .
$$

Proposition 4.1 comes from [BB18, Proposition 1.1], though in [BB18, Remark 1.2], the authors note that the formula can be obtained via some combination of results in [BdCP09].

Proposition 4.1 ([BB18]). For any $s \in \mathbb{R}$ and $v>0$, we have

$$
\mathcal{F}_{2}(s, v)=\exp \left(-\int_{s}^{\infty}(t-s) u_{\mathrm{AS}}^{2}(t ; \gamma) d t\right)
$$

and

$$
\mathcal{F}_{1}(s, v)=\sqrt{\mathcal{F}_{2}(s, 2 v)} \sqrt{1+\frac{\cosh \mu\left(s, \gamma_{2}\right)-\sqrt{\gamma_{2}} \sinh \mu\left(s, \gamma_{2}\right)-1}{2-\gamma}},
$$


where $\gamma, \mu\left(s, \gamma_{2}\right)$ and $\gamma_{2}$ are defined as in the statement of Theorem 1.\%.

Let $F_{2}(s, v):=\mathbb{E}\left[\exp \left(-v \chi^{\mathrm{Ai}}([s, \infty))\right)\right]$ be the cumulant generating function of the GUE point process. One of the major technical achievements of [CG20] is given below as Proposition 4.2, which bounds $F_{2}(s, v)$ by equating it to $\mathcal{F}_{2}(s, v)$ and then using the connection to the Painlevé II equation given by (4.1) to conduct a fine analysis.

Proposition 4.2 ([CG20, Theorem 1.7]). For all $v$ and $s$ in $\mathbb{R}$, we have

$$
F_{2}(s, v)=\mathcal{F}_{2}(s, v)=\exp \left(-\int_{-s}^{\infty}(x+s) u_{\mathrm{AS}}^{2}(x ; \gamma) d x\right)
$$

where $\gamma:=\gamma(v)=1-e^{-v}$. Furthermore, for any fixed $\delta \in\left(0, \frac{2}{5}\right)$, as $s$ goes to $\infty$,

$$
\log F_{2}\left(-s, s^{\frac{3}{2}-\delta}\right) \leq-\frac{2}{3 \pi} s^{3-\delta}+\mathcal{O}\left(s^{3-\frac{13 \delta}{11}}\right) .
$$

4.2. Fredholm Pfaffians. The Fredholm Pfaffian was first defined in [Rai00]; the definition reproduced below comes from [BBCS18].

Definition 4.3. Let $\mu$ be a reference measure on $\mathbb{R}$, and let $K(x, y)$ be a $2 \times 2$ matrix-valued skew-symmetric kernel on $\mathbb{R}^{2}$. Define

$$
J(x, y)=\mathbb{1}_{(x=y)}\left(\begin{array}{cc}
0 & 1 \\
-1 & 0
\end{array}\right), \forall x, y \in \mathbb{R} .
$$

Then the Fredholm Pfaffian of $K$ is defined by the series expansion

$$
\operatorname{Pf}(J+K)_{\mathbb{L}^{2}(\mathbb{R}, \mu)}:=1+\sum_{k=1}^{\infty} \frac{1}{k !} \int_{\mathbb{R}} \ldots \int_{\mathbb{R}} \operatorname{Pf}\left(K\left(x_{i}, x_{j}\right)_{i, j=1}^{k}\right) d \mu^{\otimes^{k}}\left(x_{1}, \ldots x_{k}\right),
$$

provided that the series converges.

Let the measure $\nu$ on $(\operatorname{Conf}(\mathbb{R}), \Sigma)$ be a Pfaffian point process on $(\mathbb{R}, \mathcal{B}(\mathbb{R}), \mu)$ with matrix kernel $K$, and let $X$ denote a random configuration with law $\nu$ (see Section 3.1 for definitions of these objects). For any measurable function $f: \mathbb{R} \rightarrow \mathbb{C}$, [Rai00, Theorem 8.2] gives the identity

$$
\mathbb{E}_{\nu}\left[\prod_{x \in X}(1+f(x))\right]=\operatorname{Pf}(J+K)_{\mathbb{L}^{2}(\mathbb{R}, f \mu)},
$$

whenever both sides converge absolutely. This identity can be applied to obtain a Fredholm Pfaffian representation for $F_{1}$. Consider the GOE point process, which we recall is a Pfafian point process on $(\mathbb{R}, \mathcal{B}(\mathbb{R}), \mu)$, where $\mu$ denotes the Lebesgue measure. Recall also that we write $\left(\mathrm{a}_{1}>\mathrm{a}_{2}>\ldots\right)$ to denote the random configuration of GOE points. For any $s \in \mathbb{R}$ and $v \geq 0$, taking $f(x):=$ $e^{-v \mathbb{1}(x \geq s)}-1$ in (4.6) yields

$$
F_{1}(s, v)=\mathbb{E}_{\mathrm{GOE}}\left[\prod_{\mathrm{a}_{i}} e^{-v \mathbb{1}\left(\mathrm{a}_{i} \geq s\right)}\right]=\operatorname{Pf}\left(J+K^{\mathrm{GOE}}\right)_{\mathbb{L}^{2}(\mathbb{R}, f \mu)},
$$

provided that the right-hand side above converges absolutely. The absolute convergence is shown in the proof of Theorem 4.4 below.

Theorem 4.4. Let $\mathcal{F}_{1}(s, v)$ denote the distribution function of the largest particle of the thinned $G O E$ point process $\mathrm{a}_{1}(\gamma)$ with parameter $\gamma:=1-e^{-v}$, where $s \in \mathbb{R}$ and $v \geq 0$. Then we have

$$
F_{1}(s, v)=\operatorname{Pf}\left(J-\gamma K^{\mathrm{GOE}}\right)_{\mathbb{L}^{2}([s, \infty), \mu)}=\mathcal{F}_{1}(s, v) .
$$

where $\mu$ denotes the Lebesgue measure. 
Proof. We begin by demonstrating the absolute convergence of the right-hand side of (4.7), which may be expanded as

$$
\begin{aligned}
& 1+\sum_{k=1}^{\infty} \frac{1}{k !} \int_{\mathbb{R}} \ldots \int_{\mathbb{R}} \operatorname{Pf}\left(K^{\mathrm{GOE}}\left(x_{i}, x_{j}\right)\right)_{i, j=1}^{k} \prod_{i=1}^{k}\left(e^{-v \mathbb{1}\left(x_{i} \geq s\right)}-1\right) d \mu^{\otimes^{k}}\left(x_{1}, \ldots, x_{k}\right) \\
& =1+\sum_{k=1}^{\infty} \frac{\left(e^{-v}-1\right)^{k}}{k !} \int_{[s, \infty)} \cdots \int_{[s, \infty)} \operatorname{Pf}\left(K^{\mathrm{GOE}}\left(x_{i}, x_{j}\right)\right)_{i, j=1}^{k} d \mu^{\otimes^{k}}\left(x_{1}, \ldots, x_{k}\right) .
\end{aligned}
$$

Observe that since $v \geq 0,\left|e^{-v}-1\right| \leq 1$. This along with the bound on $\left|\operatorname{Pf}\left(K^{\operatorname{GOE}}\left(x_{i}, x_{j}\right)\right)_{i, j=1}^{k}\right|$ given in [Lin20, Proposition 4.1(i)] allows us to compute

$$
\begin{aligned}
& \sum_{k=1}^{\infty} \frac{\left|\left(e^{-v}-1\right)^{k}\right|}{k !} \int_{[s, \infty)} \cdots \int_{[s, \infty)}\left|\operatorname{Pf}\left(K^{\mathrm{GOE}}\left(x_{i}, x_{j}\right)\right)_{i, j=1}^{k}\right| d \mu^{\otimes^{k}}\left(x_{1}, \ldots, x_{k}\right) \\
& \leq \sum_{k=1}^{\infty} \frac{k^{k / 2} C^{k}}{k !}\left(\int_{s}^{\infty} e^{-x_{i}^{3 / 2} / 3} \mathbb{1}_{\left\{x_{i} \geq 0\right\}}+(1-x)^{2} \mathbb{1}_{\{x<0\}} d \mu(x)\right)^{k} \\
& \leq \sum_{k=1}^{\infty} \frac{k^{k / 2} C_{s}^{k}}{k !}<\infty,
\end{aligned}
$$

where $C$ is a positive constant, $C_{s}$ is a positive constant depending only on $s$, and the above sum converges due to Stirling's formula. This establishes the Fredholm Pfaffian representation (4.7) of $F_{1}(s, v)$.

Let us return to the expansion of the Fredholm Pfaffian in (4.9). From the definition of $\operatorname{Pf}(A)$, we see that scaling every entry of the matrix $A$ by some constant $c$ and taking the Pfaffian is equivalent to $c^{k} \operatorname{Pf}(A)$, where $A$ is a $2 k \times 2 k$ matrix. Thus, from (4.9), we find

$$
\begin{aligned}
F_{1}(s, v) & =1+\sum_{k=1}^{\infty} \frac{(-1)^{k}}{k !} \int_{[s, \infty)} \cdots \int_{[s, \infty)} \operatorname{Pf}\left(\gamma K^{\mathrm{GOE}}\left(x_{i}, x_{j}\right)\right)_{i, j=1}^{k} d \mu^{\otimes^{k}}\left(x_{1}, \ldots, x_{k}\right) \\
& =\operatorname{Pf}\left(J-\gamma K^{\mathrm{GOE}}\right)_{\mathbb{L}^{2}([s, \infty), \mu)} .
\end{aligned}
$$

Now, recall from the first paragraph of Section 4.1 that the thinned GOE point process is Pfaffian with correlation kernel $\gamma K^{\mathrm{GOE}}$. Thus, the gap probability for the thinned GOE point process is

$$
\operatorname{Pf}\left(J-\gamma K^{\mathrm{GOE}}\right)_{\mathbb{L}^{2}([s, \infty), \mu)}=\mathbb{P}\left(\mathrm{a}_{1}(\gamma)<s\right)=: \mathcal{F}_{1}(s, v) .
$$

Substituting this into (4.11) yields (4.8) .

4.3. Proofs of Theorems $\mathbf{1 . 7}$ and 1.10. We are now ready to prove Theorem 1.7. Assuming Lemma 1.9, we will then be able to prove Theorem 1.10 as well. Lemma 1.9 is proved in Section 4.4 below.

Proof of Theorem 1.7. Equation (1.20) follows immediately from (4.2), Proposition 4.2, and Theorem 4.4.

Proof of Theorem 1.10. Fix any $\delta \in(0,2 / 5)$. Take $v$ to be $\bar{v}$ (so that $\gamma=1-e^{-\bar{v}}$ and $\gamma_{2}$ is equal to $\bar{\gamma})$ in $(1.20)$. This yields

$$
F_{1}\left(-s, \frac{1}{2} s^{3 / 2-\delta}\right)=\sqrt{F_{2}\left(-s, s^{3 / 2-\delta}\right)} \sqrt{1+\frac{\cosh \mu(-s, \bar{\gamma})-\sqrt{\bar{\gamma}} \sinh \mu(-s, \bar{\gamma})-1}{2-\gamma}} .
$$


Equation (4.4) gives the following bound as $s \rightarrow \infty$ :

$$
\sqrt{F_{2}\left(-s, s^{3 / 2-\delta}\right)} \leq \sqrt{\exp \left(-\frac{2}{3 \pi} s^{3-\delta}+\mathcal{O}\left(s^{3-\frac{13 \delta}{11}}\right)\right)}=\exp \left(-\frac{1}{3 \pi} s^{3-\delta}+\mathcal{O}\left(s^{3-\frac{13 \delta}{11}}\right)\right) .
$$

Since $\bar{\gamma} \in(0,1]$ and $2-\gamma \in[1,2)$, the second term on the right-hand side of (4.12) may be crudely bounded above as $s \rightarrow \infty$ by

$$
\sqrt{C_{1}+C_{2} \exp (|\mu(-s, \bar{\gamma})|)}
$$

for some positive constants $C_{1}$ and $C_{2}$ (independent of $s$ and $\delta$ ). From Lemma 1.9 and the above display, we find that as $s \rightarrow \infty$,

$$
\sqrt{1+\frac{\cosh \mu(-s, \bar{\gamma})-\sqrt{\bar{\gamma}} \sinh \mu(-s, \bar{\gamma})-1}{2-\gamma}}=o\left(s^{3-\delta}\right) .
$$

Substituting the bounds given by (4.13) and (4.14) into (4.12) yields (1.31).

4.4. Proof of Lemma 1.9. The proof of Lemma 1.9 is given at the end of this subsection.

Throughout this subsection, as in the statement of Lemma 1.9, we take $\delta \in(0,2 / 5)$ fixed. The parameter $s$ is taken to be positive, and we define $\bar{v}:=\bar{v}(s, \delta)$ and $\bar{\gamma}:=\bar{\gamma}(s, \delta)$ as in (1.22). Note that $\bar{\gamma}=1-e^{-2 \bar{v}}$. For some fixed constants $x_{0}>0$ and $\zeta_{0} \in(0,2 \sqrt{2} / 3)$ to be specified later, we will consider upper bounds on $u_{\mathrm{AS}}(x ; \bar{\gamma})$ over each of the following intervals of $x$ :

(1) $\left[-s,-\left(\frac{2 \sqrt{2}}{3}-\zeta_{0}\right)^{-2 / 3} s^{1-\frac{2}{3} \delta}\right]$.

(2) $\left(-\left(\frac{2 \sqrt{2}}{3}-\zeta_{0}\right)^{-2 / 3} s^{1-\frac{2}{3} \delta},-\left(\frac{2 \sqrt{2}}{3}\right)^{-2 / 3} s^{1-\frac{2}{3} \delta}\right)=: \mathbf{I}_{0}$

(3) $\left[-\left(\frac{2 \sqrt{2}}{3}\right)^{-2 / 3} s^{1-\frac{2}{3} \delta},-x_{0}\right)$

(4) $\left[-x_{0}, \infty\right)$,

Consider $\bar{\aleph}:=\aleph(x, \bar{\gamma})$ (where $\aleph(x, \gamma)$ was defined for general $\gamma \in[0,1)$ in (1.24)). The interval (1) corresponds to $\bar{\aleph} \in \bar{I}_{1}\left(\zeta_{0}\right):=\left[s^{-\delta}, \frac{2 \sqrt{2}}{3}-\zeta_{0}\right]$, which we recall from Section 1.3 is contained in the regular Boutroux region $I_{1}\left(\zeta_{0}\right):=\left(0, \frac{2 \sqrt{2}}{3}-\zeta_{0}\right)$. [Bot17, Theorem 1.10] gives an expansion for $u_{\mathrm{AS}}(x ; \gamma)$ (for general $x$ and $\gamma$ such that $\aleph \in I_{1}\left(\zeta_{0}\right)$ ) in terms of Jacobi theta functions and elliptic integrals. In [CG20, Section 6], the authors manipulate the formula from [Bot17, Theorem 1.10] into a form that is more amenable to obtaining the estimates that they seek. In our case, we only seek crude upper bounds on $u_{\mathrm{AS}}$, for which [Bot17, Theorem 1.10] and the work of [CG20, Section 6] can be combined to obtain an upper bound of order $(-x)^{1 / 2}$ on $u_{\mathrm{AS}}(x ; \gamma)$ uniformly over $\aleph \in I_{1}\left(\zeta_{0}\right)$.

Lemma 4.5. For some constant $\zeta_{0} \in(0,2 \sqrt{2} / 3)$, there exists constants $S_{0}>0$ and $C>0$ such that for all $s \geq S_{0}$ and for all $\aleph \in I_{1}\left(\zeta_{0}\right)$, we have

$$
\left|u_{\mathrm{AS}}(x ; \bar{\gamma})\right| \leq C(-x)^{1 / 2} .
$$

Proof. In what follows, we rely heavily on the notation set forth at the start of [CG20, Section 6.1] since this notation is used only in the present proof, which is rather short, we do not redefine their notation here. From equations 6.1 and 6.2 of $\left[\mathrm{CG}_{2}\right]^{6}$ (which is a reformulation of Equations 1.25 and 1.26 of [Bot17]), we see that it suffices to find appropriate bounds on

$$
\frac{1-\kappa}{\sqrt{1+\kappa^{2}}}, \quad \text { and } \quad \operatorname{cd}\left(2(-x)^{3 / 2} V K(\tilde{\kappa}), \tilde{\kappa}\right)
$$

\footnotetext{
${ }^{6}$ While [CG20, Proposition 6.1] is stated for $\zeta \in(0, \sqrt{2} / 3)$, it is written in a footnote that the result holds for all $\zeta \in(0,2 \sqrt{2} / 3)$, simply because [Bot17, Equation 1.26] holds for this wider range of $\zeta$, and [CG20, Equation 6.1,6.2] is a reformulation of [Bot17, Equations 1.25, 1.26].
} 
where we define $\tilde{\kappa}:=\frac{1-\kappa}{1+\kappa}$. It follows from [CG20, Equations 6.3,6.4] that $\kappa(\aleph)$ is bounded uniformly over bounded regions of $\aleph$, and so $\frac{1-\kappa}{\sqrt{1+\kappa^{2}}}$ is bounded uniformly over $\aleph \in I_{1}\left(\zeta_{0}\right)$.

Next, [CG20, Equation 6.9] implies that there exist $r_{0} \in[0,1)$ and $C_{1}>0$ such that for all $r \leq r_{0}$,

$$
|\operatorname{cd}(z, r)| \leq 1+C_{1} r^{2} .
$$

It is shown in the proof of Lemma 6.3 of [CG20] that $\tilde{\kappa}$ goes to zero as $\aleph$ goes to zero, and so there exists $\zeta_{0}$ sufficiently close to $2 \sqrt{2} / 3$ such that for all $\aleph \in\left(0, \frac{2 \sqrt{2}}{3}-\zeta_{0}\right]$, we have $\tilde{\kappa} \leq r_{0}$. Then from (4.17), we have

$$
\left|\operatorname{cd}\left(2(-x)^{3 / 2} V K(\tilde{\kappa}), \tilde{\kappa}\right)\right| \leq C_{2},
$$

for some $C_{2}>0$. Thus, both terms in (4.16) are bounded uniformly over $\aleph \in I_{1}\left(\zeta_{0}\right)$. Equation (4.15) then follows from [CG20, Proposition 6.1].

Taking $\zeta_{0}$ as in Lemma 4.5, it follows from (4.15) that

$$
\left|\int_{-s}^{-\left(\frac{2 \sqrt{2}}{3}-\zeta_{0}\right)^{-2 / 3} s^{1-\frac{2}{3} \delta}} u_{\mathrm{AS}}(x ; \bar{\gamma}) d x\right|=\left|\int_{\bar{\aleph} \in \bar{I}_{1}\left(\zeta_{0}\right)} u_{\mathrm{AS}}(x ; \bar{\gamma}) d x\right| \leq \mathcal{C}_{1} s^{3 / 2}
$$

for some positive constant $\mathcal{C}_{1}$.

Interval (2) corresponds to $\bar{\aleph}$ in the Stokes region $\left(\frac{2 \sqrt{2}}{3}-\zeta, \frac{2 \sqrt{2}}{3}\right)$, defined in Section 1.3. Since $\mathbf{I}_{0}$ has length of order $s^{1-\frac{2}{3} \delta}$, equation (1.27) of Conjecture 1 implies that

$$
\int_{\mathbf{I}_{0}}\left|u_{\mathrm{AS}}(x ; \bar{\gamma})\right| d x=\int_{\bar{\aleph} \in\left(\frac{2 \sqrt{2}}{3}-\zeta, \frac{2 \sqrt{2}}{3}\right)}\left|u_{\mathrm{AS}}(x ; \bar{\gamma})\right| d x=o\left(s^{3-\delta}\right) .
$$

Interval (3) corresponds to $\bar{\aleph} \in \bar{I}_{2}:=\left[\frac{2 \sqrt{2}}{3}, x_{0}^{-3 / 2} s^{\frac{3}{2}-\delta}\right.$ ), which we recall from Section 1.3 is contained in the Hastings-McLeod region $I_{2}:=\left[\frac{2 \sqrt{2}}{3}, \infty\right)$. Over this region, we have [Bot17, Theorem 1.12], reformulated below as Proposition 4.6.

Proposition 4.6 ([Bot17, Theorem 1.12 $\left.]^{7}\right)$. There exist positive constants $x_{0}, v_{0}$, and $c$ such that for all $-x \geq x_{0}, v:=-\log (1-\gamma) \geq v_{0}$, and $\aleph \in I_{2}$, we have

$$
u_{\mathrm{AS}}(x ; \bar{\gamma})=-\sqrt{-\frac{x}{2}}\left(1-\frac{e^{\frac{2}{3} \sqrt{2}(-x)^{-3 / 2}-v}}{\pi(-x)^{3 / 4} 2^{5 / 4}}+J_{2}(x, s)\right),
$$

where $\left|J_{2}(x, s)\right| \leq c(-x)^{-3 / 2}$.

Take $\gamma=\bar{\gamma}$ in Proposition 4.6 so that $v=2 \bar{v}$ (where $\bar{v}$ was defined at the start of this subsection), and let $x_{0}$ be as in the proposition. Consider $S_{0}:=S_{0}(\delta)$ such that $S_{0}^{1-\frac{2}{3} \delta}>x_{0}$ and $S_{0}^{\frac{3}{2}-\delta} \geq v_{0}$. Then for any $s \geq S_{0}$ and $x$ in interval (3) (equivalently, $\bar{\aleph} \in \bar{I}_{2}$ ), we have $-x \geq x_{0}$ and $2 \bar{v} \geq v_{0}$. Thus, the hypotheses of the proposition are satisfied, and so there exists a constant $C:=C(\delta)>0$ (independent of the choice of $\bar{\aleph} \in \bar{I}_{2}$ ) such that $\left|u_{\mathrm{AS}}(x ; \bar{\gamma})\right| \leq C(-x)^{1 / 2}$. Thus, there exists a constant $\mathcal{C}_{2}:=\mathcal{C}_{2}(\delta)>0$ such that

$$
\left|\int_{-\left(\frac{2 \sqrt{2}}{3}\right)^{-2 / 3} s^{1-\frac{2}{3} \delta}}^{-x_{0}} u_{\mathrm{AS}}(x ; \bar{\gamma})\right|=\left|\int_{\bar{\aleph} \in \bar{I}_{2}} u_{\mathrm{AS}}(x ; \bar{\gamma})\right| \leq \mathcal{C}_{2} s^{\frac{3}{2}-\delta} .
$$

\footnotetext{
${ }^{7}$ It may be helpful to match the notation of [Bot17] with ours. We have taken the parameter $f_{2}$ of [Bot17] to be 0 . For any $\gamma \in[0,1)$, the function $u(x \mid s):=u\left(x \mid\left(s_{1}, s_{2}, s_{3}\right)\right)$ of [Bot17] is equal to $u_{\mathrm{AS}}(x ; \gamma)$ in the special case $s=(-i \sqrt{\gamma}, 0, i \sqrt{\gamma})$, as stated in [Bot17, Remark 1.6]. The quantity $\varepsilon$ of [Bot17] is defined as $\operatorname{sgn}\left(\Im s_{1}\right)$, which is equal to -1 in our case. The parameter $v$ of [Bot17] is also written here as $v$. The parameter $\aleph$ of [Bot17] is defined in [Bot17, Equation 1.21] as $v(-x)^{-3 / 2}$, which, for $v=-\log (1-\gamma)$, matches our definition of $\aleph$.
} 
Finally, consider interval (4). For any fixed $x_{0}$, the integral of $u_{\mathrm{AS}}(x ; \bar{\gamma})$ over $x$ in interval (4) evaluates to a constant due to the exponential decay in (1.19). That is, there exists a positive constant $\mathcal{C}_{3}$ such that

$$
\left|\int_{-x_{0}}^{\infty} u_{\mathrm{AS}}(x ; \bar{\gamma}) d x\right|=\mathcal{C}_{3}
$$

We are now ready to prove Lemma 1.9.

Proof of Lemma 1.9. Equation (1.29) follows immediately from (4.18), (4.21), and (4.22). Equation (1.30) follows from the additional input (4.19).

\section{Proof of Theorem 1.11}

The proof of Theorem 1.11 was sketched in Section 1.2, starting from (1.17). Here, we give a complete proof. The following corollary follows from Theorem 4.4 and a less precise formulation of [BB18, Theorem 1.4], which states that $\log \mathcal{F}_{1}(-s, v)$ is given by the right-hand side of (5.1) (and thus, by Theorem 4.4, the same is true for $\left.F_{1}(-s, v)\right)$.

Corollary 5.1 ([BB18, Theorem 1.4]). Fix $\gamma \in[0,1)$ and define $v:=-\log (1-\gamma) \in[0, \infty)$. There exist positive constants $S_{0}:=S_{0}(\gamma)$ such that for all $s \geq S_{0}$, we have

$$
\log F_{1}(-s, v)=-\frac{2}{3 \pi} v s^{3 / 2}+\frac{v^{2}}{2 \pi^{2}} \log \left(8 s^{3 / 2}\right)+\mathcal{O}(1) .
$$

Proof of Theorem 1.11. Fix $\eta>0, c>0$, and $\delta \in(0,2 / 5)$. For brevity, we write $\mathcal{A}$ to denote the event

$$
\mathcal{A}:=\left\{\chi^{\mathrm{GOE}}[-s, \infty)-\mathbb{E}\left[\chi^{\mathrm{GOE}}([-s, \infty))\right] \leq-c s^{3 / 2}\right\} .
$$

For any $\lambda>0$, taking $f(x)=e^{-\lambda x}$ in Markov's inequality gives the upper-bound

$$
\begin{aligned}
\mathbb{P}(\mathcal{A}) & \leq \exp \left(-c \lambda s^{3 / 2}+\lambda \mathbb{E}\left[\chi^{\mathrm{GOE}}([-s, \infty))\right]\right) \mathbb{E}\left[\exp \left(-\lambda \chi^{\mathrm{GOE}}([-s, \infty))\right)\right] \\
& =\exp \left(-c \lambda s^{3 / 2}+\frac{2}{3 \pi} \lambda s^{3 / 2}+\lambda D_{1}(s)\right) F_{1}(-s, \lambda)
\end{aligned}
$$

where (5.2) follows from the substitution of (1.16). Taking $\lambda=2 \eta / c$ and substituting (5.1) into (5.2) yields

$$
\mathbb{P}(\mathcal{A}) \leq \exp \left(-2 \eta s^{3 / 2}+\mathcal{O}(\log s)\right) \leq \exp \left(-\eta s^{3 / 2}\right),
$$

where the last inequality holds for all $s$ sufficiently large (depending on $\eta$ and $c$ ). Thus, we have (1.32).

Now, assume Conjecture 1. Then taking $\lambda=\frac{1}{2} s^{\frac{3}{2}-\delta}$ in (5.2) gives

$$
\mathbb{P}(\mathcal{A}) \leq \exp \left(-\frac{1}{2} c s^{3-\delta}+\frac{1}{3 \pi} s^{3-\delta}+\frac{1}{2} s^{\frac{3}{2}-\delta} D_{1}(s)\right) F_{1}\left(-s, \frac{1}{2} s^{\frac{3}{2}-\delta}\right) .
$$

Substituting the bound of Theorem 1.10 into the above yields equation (1.33).

\section{Proof of Theorem 1.12}

We now prove Theorem 1.12. Our method of proof necessarily differs from the GUE case of [CG20], which benefits from the Airy kernel being a locally admissible and good trace-class operator (see [AGZ10, Section 4.2]). For such kernels, on any compact set $D \subset \mathbb{R}$, the point process can be expressed as the following sum:

$$
\chi^{\mathrm{Ai}}(D) \stackrel{(d)}{=} \sum_{i=1}^{\infty} X_{i},
$$


where the $X_{i}$ are independent Bernoulli random variables satisfying $\mathbb{P}\left(X_{i}=1\right)=1-\mathbb{P}\left(X_{i}=\right.$ $0)=\lambda_{i}^{D}$. Here, $\lambda_{i}^{D}$ are the eigenvalues of the operator $\mathbb{1}(D) K^{\mathrm{Ai}} \mathbb{1}(D)$. An application of Bennet's concentration inequality yields the desired upper large deviations bound on $\chi^{\mathrm{Ai}}$.

Pfaffian point processes possess matrix-valued kernels (see Section 3), and while [Kar14] describes a such class of kernels whose corresponding Pfaffian point processes can be expressed as a sum of Bernoulli random variables, no such result is known for the GOE point process. Instead, we estimate $\chi^{\mathrm{GOE}}$ on intervals by carefully analyzing the closest GOE points to the boundary of the interval. The result is the exponential upper bound (1.34), which suffices to establish (2.10), which in turn gives the lower bound (1.11) on the half-space KPZ tail.

Proof of Theorem 1.12. Throughout this proof, we write $\chi:=\chi^{\mathrm{GOE}}$ for brevity. Fix $c>0$ and $\delta \in(0,2 / 5)$. In what follows, we will write $\hat{c}:=\hat{c}(c)$ to denote a positive constant depending only on the parameter $c$ whose value may change from line to line. We first consider $\mathfrak{B}_{k}(\ell)$ for $k \geq 2$.

As usual, let $\left(\mathrm{a}_{1}>\mathrm{a}_{2}>\ldots\right)$ denote the GOE point process, and let $\left(\lambda_{1}<\lambda_{2}<\ldots\right)$ denote the eigenvalues of the Airy operator. Define

$$
\begin{aligned}
& m_{1}:=\sup \left\{m: \mathrm{a}_{m} \geq-(k-1) \ell\right\}, \quad m_{2}:=\sup \left\{m: \mathrm{a}_{m} \geq-k \ell\right\}, \text { and } \\
& k_{1}:=\sup \left\{n:-\lambda_{n} \geq-(k-1) \ell\right\}, \quad k_{2}:=\sup \left\{n:-\lambda_{n} \geq-k \ell\right\} .
\end{aligned}
$$

Note that $\chi\left(\mathfrak{B}_{k}(\ell)\right)=m_{2}-m_{1}$. Theorem 1.6 gives us

$$
\mathbb{E}\left[\chi\left(\mathfrak{B}_{k}(\ell)\right)\right]=\frac{2}{3 \pi}\left(k^{3 / 2}-(k-1)^{3 / 2}\right) \ell^{3 / 2}+f_{1},
$$

where $\left.f_{1}:=f_{1}(k, \ell)=D_{1}(k \ell)-D_{1}((k-1) \ell)\right)$; note that $f_{1}$ is bounded in $k$ and $\ell$. By Taylor's theorem, we have

$$
k^{3 / 2}-(k-1)^{3 / 2}=\frac{3}{2}(k-1)^{1 / 2}+R_{k},
$$

where $0<R_{k} \leq \frac{3}{4}$. By Corollary 3.5, we have

$$
\mathbb{E}\left[\chi\left(\mathfrak{B}_{k}(\ell)\right)\right]=k_{2}-k_{1}+f_{2},
$$

where $f_{2}:=f_{2}(k, \ell)$ is bounded in $k$ and $\ell$. Define the positive constant

$$
\mathfrak{c}_{k}:=\mathfrak{c}_{k}(c)=c\left(\frac{1}{\pi}(k-1)^{1 / 2}+\frac{2}{3 \pi} R_{k}\right)^{-1},
$$

which is bounded above uniformly in $k$, and satisfies

$$
\mathfrak{c}_{k} \geq \hat{c} k^{-1 / 2} .
$$

Then substituting (6.2) and (6.3) into (6.1) yields

$$
c \ell^{3 / 2}=\mathfrak{c}_{k}\left(k_{2}-k_{1}\right)-f_{3},
$$

where $f_{3}:=f_{3}(k, \ell)$ is bounded in $k$ and $\ell$. The above display along with the relation $\chi\left(\mathfrak{B}_{k}(\ell)\right)=$ $m_{2}-m_{1}$ gives

$$
\left\{\chi\left(\mathfrak{B}_{k}(\ell)\right)-\mathbb{E}\left[\chi\left(\mathfrak{B}_{k}(\ell)\right] \geq c \ell^{3 / 2}\right\}=\left\{m_{2}-m_{1} \geq\left(1+\mathfrak{c}_{k}\right)\left(k_{2}-k_{1}\right)+f_{3}\right\} .\right.
$$

It follows that the event $\left\{\chi\left(\mathfrak{B}_{k}(\ell)\right)-\mathbb{E}\left[\chi\left(\mathfrak{B}_{k}(\ell)\right] \geq c \ell^{3 / 2}\right\}\right.$ is contained in the event

$$
\left\{m_{2} \geq k_{2}+\frac{\mathfrak{c}_{k}}{2}\left(k_{2}-k_{1}\right)+f_{3}\right\} \cup\left\{m_{1} \leq k_{1}-\frac{\mathfrak{c}_{k}}{2}\left(k_{2}-k_{1}\right)\right\} .
$$

The next two claims provide an upper-bound on each of the events in the above union. 
Claim 6.1. There exist positive constants $\bar{c}:=\bar{c}(c), \kappa:=\kappa(c, \delta)$, and $\ell_{0}:=\ell_{0}(c, \delta)$ such that for all $\ell \geq \ell_{0}$, we have

$$
\mathbb{P}\left(m_{2} \geq k_{2}+\frac{\mathfrak{c}_{k}}{2}\left(k_{2}-k_{1}\right)+f_{3}\right) \leq \kappa \exp \left(-\kappa(\bar{c} \ell)^{1-\delta}\right) .
$$

Proof of Claim 6.1. Since $-\mathrm{a}_{m_{2}} \leq k \ell$, Theorem 1.5 yields

$$
(1-\varepsilon) \lambda_{m_{2}}-k \ell \leq C_{\varepsilon}^{\mathrm{GOE}},
$$

for any $\varepsilon \in(0,1)$. Let $k_{3}:=k_{2}+\frac{\mathfrak{c}_{k}}{2}\left(k_{2}-k_{1}\right)+f_{3}$. Since $\lambda_{i}<\lambda_{j}$ if and only if $i<j$, the previous display gives us

$$
\left\{m_{2} \geq k_{3}\right\} \subseteq\left\{(1-\varepsilon) \lambda_{k_{3}}-k \ell \leq C_{\varepsilon}^{\mathrm{GOE}}\right\},
$$

for any $\varepsilon \in(0,1)$. Corollary 3.5 allows us to write

$$
\begin{aligned}
& k_{1}=\frac{2}{3 \pi}((k-1) \ell)^{3 / 2}+C_{1}((k-1) \ell), \text { and } \\
& k_{2}=\frac{2}{3 \pi}(k \ell)^{3 / 2}+C_{2}(k \ell),
\end{aligned}
$$

where $\sup _{x>0}\left\{\left|C_{1}(x)\right|,\left|C_{2}(x)\right|\right\}<1$. Then, from Proposition 3.4 and the definition of $k_{3}$, we compute

$$
\begin{aligned}
\lambda_{k_{3}} & =\left((k \ell)^{3 / 2}+\frac{\mathfrak{c}_{k}}{2}\left((k \ell)^{3 / 2}-((k-1) \ell)^{3 / 2}\right)+f_{4}\right)^{2 / 3} \\
& =(k \ell)\left(1+\frac{\mathfrak{c}_{k}}{2}\left(1-\left(\frac{k-1}{k}\right)^{3 / 2}\right)+(k \ell)^{-3 / 2} f_{4}\right)^{2 / 3},
\end{aligned}
$$

where $f_{4}:=f_{4}(k, \ell)$ is bounded in $k$ and $\ell$. Since the function $g(x):=x^{2 / 3}$ is an increasing function in $x,(6.11)$ gives us

$$
\lambda_{k_{3}} \geq(k \ell)\left(1+\frac{\mathfrak{c}_{k}}{4}\right)^{2 / 3},
$$

for all $\ell \geq 1$ (and recall that we have fixed $k \geq 2$ ). Substituting (6.12) into (6.8), we find

$$
\left\{m_{2} \geq k_{3}\right\} \subseteq\left\{C_{\varepsilon}^{\mathrm{GOE}} \geq k \ell\left((1-\varepsilon)\left(1+\frac{\mathfrak{c}_{k}}{4}\right)^{2 / 3}-1\right)\right\} .
$$

We now show that there exists some $\varepsilon \in(0,1)$ such that $k\left((1-\varepsilon)\left(1+\frac{\mathfrak{c}_{k}}{4}\right)^{2 / 3}-1\right)$ can be bounded below by a positive constant $\bar{c}:=\bar{c}(c)$ uniformly in $k \in \mathbb{Z}_{\geq 2}$. Define

$$
\hat{\mathfrak{c}}_{k}:=\hat{\mathfrak{c}}_{k}(\varepsilon)=\left((1-\varepsilon)\left(1+\frac{\mathfrak{c}_{k}}{4}\right)^{2 / 3}-1\right) .
$$

It is clear that from (6.4) that for any fixed $k$, there exists $\varepsilon>0$ such that $\hat{\mathfrak{c}}_{k}>0$. Thus, we need only consider $k$ arbitrarily large. We show that there exists a positive constant $K:=K(c)$ such that for all $k \geq K(c)$, there exists $\varepsilon:=\varepsilon(k, c)>0$ such that $\hat{\mathfrak{c}}_{k}(\varepsilon)=k^{-1}$. Towards this end, using (6.4), we find the lower-bound

$$
\varepsilon=1-\frac{1+\hat{\mathfrak{c}}_{k}}{\left(1+\frac{\mathfrak{c}_{k}}{4}\right)^{2 / 3}} \geq 1-\frac{1+\hat{\mathfrak{c}}_{k}}{\left(1+\hat{c}(k-1)^{-1 / 2}\right)^{2 / 3}} .
$$

That $\varepsilon<1$ is trivial. Thus, it suffices to show that there exists a positive constant $K:=K(c)$ such that

$$
k^{-1}<\left(1+\hat{c}(k-1)^{-1 / 2}\right)^{2 / 3}-1
$$


for all $k \geq K$ (for then it will follow that there exists $\varepsilon(k, c) \in(0,1)$ such that $\hat{c}_{k}=k^{-1}$, for all $k \geq K)$. Let $K:=K(c)$ be large enough such that $\hat{c}(k-1)^{-1 / 2}<1$ for all $k \geq K$. Then, by Taylor's theorem, we have

$$
\left(1+\hat{c}(k-1)^{-1 / 2}\right)^{2 / 3}-1=\frac{2}{3} \hat{c}(k-1)^{-1 / 2}+\mathcal{O}\left(k^{-1}\right) \geq \hat{c}(k-1)^{-1 / 2},
$$

where the last inequality holds for $K:=K(c)$ large enough and all $k \geq K$ (and the $\hat{c}$ on the right-most side differs from the other $\hat{c}$ ). Now, choose $K$ large enough such that, for all $k \geq K$, we have $k^{-1}<\hat{c}(k-1)^{-1 / 2}$. Then, from (6.16), it follows that (6.15) holds. Thus, we may take

$$
\bar{c}=\min \left\{1, \min _{k \leq K} k \mathfrak{c}_{k}\right\},
$$

which depends only on $c$.

Now, let $k_{0}:=k_{0}(c) \in \mathbb{Z}_{\geq 1}$ and $\varepsilon_{0}:=\varepsilon_{0}(c) \in(0,1)$ be such that $\bar{c}=c_{k_{0}}\left(\varepsilon_{0}\right)$. Thus, from (6.13), we have

$$
\left\{m_{2} \geq k_{3}\right\} \subseteq\left\{C_{\varepsilon_{0}}^{\mathrm{GOE}} \geq \bar{c}\right\}
$$

Equation (6.17) and Theorem 1.5 then give the final result: there exist positive constants $\kappa:=\kappa(c, \delta)$ and $L_{0}:=L_{0}(c, \delta)$ such that for all $\ell \geq \ell_{0}$, we have

$$
\mathbb{P}\left(m_{2} \geq k_{2}+\frac{\mathfrak{c}_{k}}{2}\left(k_{2}-k_{1}\right)\right) \leq \mathbb{P}\left(C_{\varepsilon_{0}}^{\mathrm{GOE}} \geq \bar{c} \ell\right) \leq \kappa \exp \left(-\kappa(\bar{c} \ell)^{1-\delta}\right) .
$$

This concludes the proof of Claim 6.1.

Claim 6.2. For any $\eta>0$, there exists a positive constant $\bar{L}_{0}:=\bar{L}_{0}(c, \eta)$ such that for all $\ell \geq \bar{L}_{0}$, we have

$$
\mathbb{P}\left(m_{1} \leq k_{1}-\frac{\mathfrak{c}_{k}}{2}\left(k_{2}-k_{1}\right)\right) \leq \exp \left(-\eta \ell^{3 / 2}\right) .
$$

Proof of Claim 6.2. Fix $\eta>0$. Let the left-hand side of (6.18) be denoted by $\mathcal{P}$. By definition of $m_{1}$, we have $m_{1}=\chi(-(k-1) \ell, \infty)$. Corollary 3.5 gives the expression

$$
m_{1}-k_{1}=\chi(-(k-1) \ell, \infty)-\mathbb{E}[\chi(-(k-1) \ell, \infty)]+g_{1}
$$

where $g_{1}:=g_{1}(k, \ell)$ is bounded in $k$ and $\ell$. This expression allows us to write $\mathcal{P}$ as

$$
\mathcal{P}=\mathbb{P}\left(\chi(-(k-1) \ell, \infty)-\mathbb{E}[\chi(-(k-1) \ell, \infty)] \leq-\frac{\mathfrak{c}_{k}}{2}\left(k_{2}-k_{1}\right)+g_{1}\right) .
$$

From equations (6.9), (6.10), and (6.2), we may write

$$
k_{2}-k_{1}=\frac{2}{3 \pi}\left(\frac{3}{2}(k-1)^{1 / 2}+R_{k}\right) \ell^{3 / 2}+g_{2},
$$

where $g_{2}:=g_{2}(k, \ell)$ is bounded in $k$ and $\ell$. The above along with (6.4) yield the bound

$$
-\frac{\mathfrak{c}_{k}}{2}\left(k_{2}-k_{1}\right)+g_{1} \leq-\hat{c} k^{-1 / 2}\left(\frac{3}{2}(k-1)^{1 / 2}+R_{k}\right) \ell^{3 / 2}+g_{3} \leq-\bar{C} \ell^{3 / 2},
$$

where $g_{3}:=g_{3}(k, \ell)$ is bounded in $k$ and $\ell$ and $\bar{C}:=\bar{C}(c)$ is a positive constant; and the last inequality holds for all $\ell \geq \bar{L}_{0}$, where $\bar{L}_{0}:=\bar{L}_{0}(c)$ is sufficiently large. Substituting (6.20) into the right-hand side of (6.19) yields

$$
\mathcal{P} \leq \mathbb{P}\left(\chi(-(k-1) \ell, \infty)-\mathbb{E}[\chi(-(k-1) \ell, \infty)] \leq-\bar{C} \ell^{3 / 2}\right) .
$$

We may now apply equation (1.32) of Theorem 1.11: in the notation of this theorem, we take $c$ to be $\bar{C}, s$ to be $\ell$, and $\eta$ to be the same $\eta$ here. Then there exists a positive constant $\bar{L}_{0}:=\bar{L}_{0}(c, \eta)$ such that for all $\ell \geq \bar{L}_{0}$, we have $\mathcal{P} \leq \exp \left(-\eta \ell^{3 / 2}\right)$ as desired. This concludes the proof of Claim 6.2. 
We are now ready to conclude the proof of Theorem 1.12. Define

$$
\overline{\mathcal{P}}:=\mathbb{P}\left(\chi\left(\mathfrak{B}_{k}(\ell)\right)-\mathbb{E}\left[\chi\left(\mathfrak{B}_{k}(\ell)\right] \geq c \ell^{3 / 2}\right) .\right.
$$

From (6.6), we have

$$
\overline{\mathcal{P}} \leq \mathbb{P}\left(m_{2} \geq k_{2}+\frac{\mathfrak{c}_{k}}{2}\left(k_{2}-k_{1}\right)+f_{3}\right)+\mathbb{P}\left(m_{1} \leq k_{1}-\frac{\mathfrak{c}_{k}}{2}\left(k_{2}-k_{1}\right)\right) .
$$

Substituting the bounds obtained in (6.7) and (6.18) gives

$$
\overline{\mathcal{P}} \leq \kappa \exp \left(-\kappa(\bar{c} \ell)^{1-\delta}\right)+\exp \left(-\eta \ell^{3 / 2}\right) \leq \exp \left(-\mathcal{C} \ell^{1-\delta}\right)
$$

where the first inequality holds for any fixed $\eta>0$ and all $\ell \geq L_{0}$, where $L_{0}:=L_{0}(c, \delta, \eta)$ is greater than or equal to $\max \left\{\ell_{0}, \bar{L}_{0}\right\}$. Fixing $\eta$, the second inequality above holds for a (possibly larger) $L_{0}$ and another positive constant $\mathcal{C}:=\mathcal{C}(c, \delta)$. This concludes the proof of the result for $k \geq 2$.

Now, if $k=1$, take $m_{2}$ defined as in the $k \geq 2$ case. Then (6.5) holds with $m_{1}=0$, i.e., we have

$$
\begin{aligned}
\left\{\chi\left(\mathfrak{B}_{k}(\ell)\right)-\mathbb{E}\left[\chi\left(\mathfrak{B}_{k}(\ell)\right] \geq c \ell^{3 / 2}\right\}\right. & =\left\{\chi\left(\mathfrak{B}_{k}(\ell)\right)-\mathbb{E}\left[\chi\left(\mathfrak{B}_{k}(\ell)\right] \geq \mathfrak{c}_{k} \mathbb{E}\left[\chi\left(\mathfrak{B}_{k}(\ell)\right]+f_{3}\right\}\right.\right. \\
& =\left\{m_{2} \geq\left(1+\mathfrak{c}_{k}\right)\left(k_{2}-k_{1}\right)+f_{3}\right\} .
\end{aligned}
$$

Then (6.7) finishes the proof for the $k=1$ case.

\section{Proof of Proposition 2.2}

In this section, we prove Proposition 2.2, thus completing our proof of Theorem 1.4. Here, we follow closely the method of [CG20, Section 5]; indeed, many of the computations done there are adapted here to our case.

Before proceeding, we recall a result describing the tail behavior of $\mathrm{a}_{1}$, which follows the GOE Tracy-Widom distribution (see [TW96]). The following proposition is a much simplified version of a result of [BBD08], where the authors extract precise asymptotics up to the third order (prior, the asymptotic behavior had been known by studying the asymptotics of the solutions of the Painlevé II equation).

Proposition 7.1 ([BBD08]). Let $\mathrm{a}_{1}$ denote the top particle in the GOE point process. Then

$$
\mathbb{P}\left(\mathrm{a}_{1}<-s\right)=\exp \left(-\frac{1}{24} s^{3}(1+o(1))\right) .
$$

7.1. Proof of the upper bound, equations (2.11) and (2.12). Recall that we defined in (2.9)

$$
J_{s}(x):=\frac{1}{2} \log \left(1+\exp \left(T^{1 / 3}(x+s)\right), \text { and } I_{s}(x):=\exp \left(-J_{s}(x)\right)\right.
$$

We will establish an upper bound on $\mathbb{E}_{\mathrm{GOE}}\left[\prod_{k=1}^{\infty} I_{s}\left(\mathrm{a}_{k}\right)\right]$ by deriving a lower bound on $\sum_{k=1}^{\infty} J_{s}\left(\mathrm{a}_{k}\right)$. To this end, we denote $D_{k}:=\left(-\lambda_{k}-\mathrm{a}_{k}\right)_{+}$, where we write $x_{+}:=\max \{x, 0\}$ for any $x \in \mathbb{R}$.

Lemma 7.2. Fix $\varepsilon \in(0,1 / 3)$. Define $\theta_{0}:=\left\lfloor 2 s^{3 / 2} / 3 \pi\right\rfloor$. There exist positive constants $S_{0}:=S_{0}(\varepsilon)$ and $R$ such that for all $s \geq S_{0}$ and for all $T \geq 0$,

$$
\sum_{k=1}^{\infty} J_{s}\left(\mathrm{a}_{k}\right) \geq \frac{1}{2} T^{1 / 3}\left(\frac{4 s^{5 / 2}}{15 \pi}(1-8 \varepsilon)-\sum_{k=1}^{\theta_{0}} D_{k}-R\right) .
$$

Proof. We compute

$$
\sum_{k=1}^{\infty} J_{s}\left(\mathrm{a}_{k}\right)=\sum_{k=1}^{\infty} J_{s}\left(-\lambda_{k}-D_{k}+\left(-\lambda_{k}-\mathrm{a}_{k}\right)_{-}\right) \geq \sum_{k=1}^{\infty} J_{s}\left(-\lambda_{k}-D_{k}\right),
$$


where the inequality comes from the fact that $J_{s}(x)$ is a monotonically increasing function. We now divide the sum on the right-hand side of (7.3) into three ranges: $\left[1, \theta_{1}\right],\left(\theta_{1}, \theta_{2}\right)$, and $\left[\theta_{2}, \infty\right)$, where we define

$$
\mathcal{K}:=\sup _{n \geq 1}\{|n \mathcal{R}(n)|\}, \theta_{1}:=\lceil 4 \mathcal{K}\rceil, \theta_{2}:=\left\lceil\frac{2 s^{3 / 2}}{3 \pi}+\frac{1}{2}\right\rceil .
$$

Here, we recall $\mathcal{R}(n)$ from Proposition 3.4, and note that $\mathcal{K}<\infty$. Note further that $\theta_{1}$ does not depend on our choice of $s$, but $\theta_{2}$ does, and so we can choose $s$ large enough so that $\theta_{1}<\theta_{2}$. Thus, we take $S_{0}$ large enough such that for all $s \geq S_{0}$, we have $\theta_{1}<\theta_{2}$. The following two claims establish appropriate lower-bounds on the sum of $J_{s}\left(-\lambda_{k}-D_{k}\right)$ over the first two ranges of $k$.

Claim 7.3. For all $s \geq 0$,

$$
\sum_{k=1}^{\theta_{1}} J_{s}\left(-\lambda_{k}-D_{k}\right) \geq \frac{1}{2} T^{1 / 3}\left(\theta_{1} s-\theta_{1}\left(\frac{3 \pi(4 \mathcal{K}+1)}{2}\right)^{2 / 3}-\sum_{k=1}^{\theta_{1}} D_{k}\right) .
$$

Proof of Claim 7.3. Note that for any $a \in \mathbb{R}$, we have $\log (1+\exp (a)) \geq a$. It follows that $J_{s}(x) \geq$ $\frac{1}{2} T^{1 / 3}(s+x)$. Using this and the fact that the $\lambda_{k}$ increase in $k$, we have

$$
\sum_{k=1}^{\theta_{1}} J_{s}\left(-\lambda_{k}-D_{k}\right) \geq \frac{1}{2} T^{1 / 3} \sum_{k=1}^{\theta_{1}} s-\lambda_{k}-D_{k} \geq \frac{1}{2} T^{1 / 3}\left(\theta_{1}\left(s-\lambda_{\theta_{1}}\right)-\sum_{k=1}^{\theta_{1}} D_{k}\right) .
$$

From Proposition 3.4,

$$
\lambda_{\theta_{1}} \leq\left(\frac{3 \pi\left(\theta_{1}-\frac{1}{4}+\frac{\mathcal{K}}{\theta_{1}}\right)}{2}\right)^{2 / 3} .
$$

Since $\theta_{1}-\frac{1}{4}+\frac{\mathcal{K}}{\theta_{1}} \leq 4 \mathcal{K}+1,(7.5)$ follows. This concludes the proof of Claim 7.3.

Claim 7.4. There exists a positive constant $S_{0}:=S_{0}(\varepsilon)$ such that for all $s \geq S_{0}$,

$$
\sum_{k=\theta_{1}+1}^{\theta_{2}-1} J_{s}\left(-\lambda_{k}-D_{k}\right) \geq \frac{1}{2} T^{1 / 3}\left(\frac{4 s^{5 / 2}}{15 \pi}(1-3 \varepsilon)-\left(\theta_{1}+1\right) s-\sum_{k=\theta_{1}+1}^{\theta_{2}-1} D_{k}\right) .
$$

Proof of Claim 7.4. Using similar bounds as in (7.6), along with the fact that $\lambda_{k} \leq(3 \pi k / 2)^{2 / 3}$ for all $k>\theta_{1}$, we find

$$
\sum_{k=\theta_{1}+1}^{\theta_{2}-1} J_{s}\left(-\lambda_{k}-D_{k}\right) \geq \frac{1}{2} T^{1 / 3} \sum_{k=\theta_{1}+1}^{\theta_{2}-1}\left(s-\left(\frac{3 \pi k}{2}\right)^{2 / 3}-D_{k}\right) .
$$

We now bound the following sum with an integral, as the summands are decreasing in $k$ :

$$
\begin{aligned}
\sum_{k=\theta_{1}+1}^{\theta_{2}-1}\left(s-\left(\frac{3 \pi k}{2}\right)^{2 / 3}\right) & \geq \int_{\theta_{1}+1}^{\theta_{2}-1} s-\left(\frac{3 \pi z}{2}\right)^{2 / 3} d z \\
& \geq \int_{0}^{\theta_{2}-1} s-\left(\frac{3 \pi z}{2}\right)^{2 / 3} d z-\left(\theta_{1}+1\right) s \\
& =\left(\theta_{2}-1\right)\left(s-\frac{3}{5}\left(\frac{3 \pi}{2}\right)^{2 / 3}\left(\theta_{2}-1\right)^{2 / 3}\right)-\left(\theta_{1}+1\right) s .
\end{aligned}
$$


Note that $\theta_{2}-1 \geq \frac{2 s^{3 / 2}}{3 \pi}-\frac{1}{2}$, and thus for $s \geq\left(\frac{3 \pi}{4 \varepsilon}\right)^{2 / 3}$, we have

$$
(1-\varepsilon) \frac{2 s^{3 / 2}}{3 \pi} \leq \theta_{2}-1 \leq \frac{2 s^{3 / 2}}{3 \pi}+1 \text {. }
$$

Substituting this bound into (7.9) and then substituting into (7.8) leads to (7.7). This concludes the proof of Claim 7.4.

Returning to the proof of Lemma 7.2, we substitute the bounds given by (7.5), (7.7), and $\sum_{k=\theta_{2}}^{\infty} J_{s}\left(-\lambda_{k}-D_{k}\right) \geq 0$ into (7.3) to obtain

$$
\sum_{k=1}^{\infty} J_{s}\left(\mathrm{a}_{k}\right) \geq \frac{1}{2} T^{1 / 3}\left[\frac{4 s^{5 / 2}}{15 \pi}(1-3 \varepsilon)-\theta_{1}\left(\frac{3 \pi(4 \mathcal{K}+1)}{2}\right)^{2 / 3}-s-\sum_{k=1}^{\theta_{2}-1} D_{k}\right] .
$$

Recalling $\theta_{1}:=\lceil 4 \mathcal{K}\rceil$, we note that $\theta_{1}(3 \pi(4 \mathcal{K}+1) / 2)^{2 / 3}$ is a constant which can be replaced by a large constant $R>0$. Finally, for sufficiently large $s \geq S_{0}$, we have $s \leq \frac{4 \varepsilon s^{5 / 2}}{3 \pi}$, and thus we may make this replacement in (7.10) to obtain (7.2). This completes the proof of Lemma 7.2.

Proof of (2.11) and (2.12) in Proposition 2.2. From (7.2), we have

$$
\prod_{k=1}^{\infty} I_{s}\left(\mathrm{a}_{k}\right)=\exp \left(-\sum_{k=1}^{\infty} J_{s}\left(\mathrm{a}_{k}\right)\right) \leq \exp \left(-\frac{1}{2} T^{1 / 3}\left(\frac{4 s^{5 / 2}}{15 \pi}(1-8 \varepsilon)-\sum_{k=1}^{\theta_{0}} D_{k}-R\right)\right),
$$

for all $s \geq S_{0}$ and for all $T \geq 0$. Note that for $S_{0}$ sufficiently large, we have

$$
\varepsilon s \theta_{0}+R \leq \frac{4 s^{5 / 2}}{15 \pi}\left(\frac{5}{2} \varepsilon+\frac{15 \pi R}{4 s^{5 / 2}}\right)<\frac{4 s^{5 / 2}}{15 \pi}(3 \varepsilon)
$$

for all $s \geq S_{0}$. Define $\mathcal{S}_{\theta_{0}}:=\sum_{k=1}^{\theta_{0}} D_{k}$. Then (7.11) and (7.12) yield

$$
\mathbb{1}\left(\mathcal{S}_{\theta_{0}}<\varepsilon s \theta_{0}\right) \prod_{k=1}^{\infty} I_{s}\left(\mathrm{a}_{k}\right) \leq \exp \left(-T^{1 / 3} \frac{2 s^{5 / 2}}{15 \pi}(1-11 \varepsilon)\right) .
$$

On the other hand, if $S_{\theta_{0}} \geq \varepsilon s \theta_{0}$, then there exists at least one $k \in\left[1, \theta_{0}\right] \cap \mathbb{Z}$ such that $D_{k}>\varepsilon s$. Thus, $\left\{S_{\theta_{0}} \geq \varepsilon s \theta_{0}\right\} \subset \bigcup_{k=1}^{\theta_{0}}\left\{D_{k} \geq \varepsilon s\right\}$. It follows that

$$
\begin{aligned}
\mathbb{E}_{\mathrm{GOE}}\left[\prod_{k=1}^{\infty} I_{s}\left(\mathrm{a}_{k}\right)\right] & =\mathbb{E}\left[\mathbb{1}\left(\mathcal{S}_{\theta_{0}}<\varepsilon s \theta_{0}\right) \prod_{k=1}^{\infty} I_{s}\left(\mathrm{a}_{k}\right)\right]+\mathbb{E}\left[\mathbb{1}\left(S_{\theta_{0}} \geq \varepsilon s \theta_{0}\right) \prod_{k=1}^{\infty} I_{s}\left(\mathrm{a}_{k}\right)\right] \\
& \leq \exp \left(-T^{1 / 3} \frac{2 s^{5 / 2}}{15 \pi}(1-11 \varepsilon)\right)+\mathbb{E}\left[\mathbb{1}\left(\bigcup_{k=1}^{\theta_{0}}\left\{D_{k} \geq \varepsilon s\right\}\right) \prod_{k=1}^{\infty} I_{s}\left(\mathrm{a}_{k}\right)\right] .
\end{aligned}
$$

We split the indicator function as

$$
\mathbb{1}\left(\bigcup_{k=1}^{\theta_{0}}\left\{D_{k} \geq \varepsilon s\right\}\right) \leq \mathbb{1}\left(\bigcup_{k=1}^{\theta_{0}}\left\{D_{k} \geq \varepsilon s\right\} \cap\left\{\mathrm{a}_{1} \geq-(1-\varepsilon) s\right\}\right)+\mathbb{1}\left(\mathrm{a}_{1} \leq-(1-\varepsilon) s\right) .
$$

Since $I_{s}\left(\mathrm{a}_{k}\right) \leq 1$ for all $k \in \mathbb{Z}_{\geq 1}$, we have that when $\mathrm{a}_{1} \geq-(1-\varepsilon) s$,

$$
\prod_{k=1}^{\infty} I_{s}\left(\mathrm{a}_{k}\right) \leq I_{s}\left(\mathrm{a}_{1}\right) \leq \frac{1}{\sqrt{1+\exp \left(T^{1 / 3}\left(s+\mathrm{a}_{1}\right)\right)}} \leq \exp \left(-\frac{1}{2} \varepsilon s T^{1 / 3}\right) .
$$


Substituting (7.15) and (7.16) into (7.14) gives

$$
\begin{aligned}
\mathbb{E}_{\mathrm{GOE}}\left[\prod_{k=1}^{\infty} I_{s}\left(\mathrm{a}_{k}\right)\right] & \leq \exp \left(-\frac{2(1-11 \varepsilon)}{15 \pi} T^{1 / 3} s^{5 / 2}\right)+\exp \left(-\frac{1}{2} \varepsilon s T^{1 / 3}\right) \mathbb{P}\left(\bigcup_{k=1}^{\theta_{0}}\left\{D_{k} \geq \varepsilon s\right\}\right) \\
& +\mathbb{P}\left(\mathrm{a}_{1} \leq-(1-\varepsilon) s\right) .
\end{aligned}
$$

Using (7.1), we have

$$
\mathbb{P}\left(\mathrm{a}_{1} \leq-(1-\varepsilon) s\right)=\exp \left(-(1-\varepsilon)^{3} \frac{s^{3}}{24}(1+o(1))\right) \leq \exp \left(-\frac{s^{3}}{24}(1-C \varepsilon)\right),
$$

for some constant $C>0$ and all $s$ sufficiently large. Now, taking $C=\max \{C, 11\}$ and using Lemma 7.5, we obtain both (2.11) and (2.12).

Lemma 7.5. Fix $\eta>0, \varepsilon \in(0,1 / 3)$, and $\delta \in(0,1 / 4)$. Then there exist positive constants $S_{0}:=S_{0}(\eta, \varepsilon, \delta)>0$ and $K_{1}:=K_{1}(\varepsilon, \delta)>0$ such that the following holds for all $s \geq S_{0}$. Divide the interval $[-s, 0]$ into $\left\lceil 2 \varepsilon^{-1}\right\rceil+1$ segments $\mathcal{Q}_{i}:=[-j \varepsilon s / 2,-(j-1) \varepsilon s / 2)$ for $j=1, \ldots,\left\lceil 2 \varepsilon^{-1}\right\rceil+1$. Denote the left and right endpoints of $\mathcal{Q}_{j}$ by $p_{j}$ and $q_{j}$ respectively. Define $k_{j}:=\#\left\{k:-\lambda_{k} \geq q_{j}\right\}$, where $\left(\lambda_{1}<\lambda_{2}<\ldots\right)$ denote the Airy operator eigenvalues. Then (recalling $\theta_{0}=\left\lfloor 2 s^{3 / 2} / 3 \pi\right\rfloor$ ), for all $j \in\left\{1, \ldots,\left\lceil 2 \varepsilon^{-1}\right\rceil+1\right\}$, we have

$$
\begin{aligned}
\mathbb{P}\left(\mathrm{a}_{k_{j}} \leq p_{j}\right) & \leq \exp \left(-\eta s^{3 / 2}\right), \text { and } \\
\mathbb{P}\left(\bigcup_{k=1}^{\theta_{0}}\left\{D_{k} \geq \varepsilon s\right\}\right) & \leq \exp \left(-\eta s^{3 / 2}\right),
\end{aligned}
$$

and, assuming Conjecture 1, we have

$$
\begin{aligned}
\mathbb{P}\left(\mathrm{a}_{k_{j}} \leq p_{j}\right) & \leq \exp \left(-K_{1} s^{3-\delta}\right), \text { and } \\
\mathbb{P}\left(\bigcup_{k=1}^{\theta_{0}}\left\{D_{k} \geq \varepsilon s\right\}\right) & \leq \exp \left(-K_{1} s^{3-\delta}\right) .
\end{aligned}
$$

Proof. If $\mathrm{a}_{k_{j}} \leq p_{j}$, then

$$
\chi^{\mathrm{GOE}}([-j \varepsilon s / 2, \infty)) \leq k_{j} .
$$

Corollary 3.5 gives us the following expressions:

$$
\begin{aligned}
k_{j} & =\frac{2}{3 \pi}(j \varepsilon s / 2)^{3 / 2}+C_{1}(j \varepsilon s / 2), \text { and } \\
\mathbb{E}\left[\chi^{\mathrm{GOE}}([-j \varepsilon s / 2, \infty))\right] & =\frac{2}{3 \pi}(j \varepsilon s / 2)^{3 / 2}+C_{2}(j \varepsilon s / 2),
\end{aligned}
$$

where $M^{\prime}:=\sup _{x \geq 0}\left\{\left|C_{1}(x)\right|,\left|C_{2}(x)\right|\right\}<\infty$. It follows from (7.23)-(7.25) that if $\mathrm{a}_{k_{j}} \leq p_{j}$, then

$$
\begin{aligned}
\chi^{\mathrm{GOE}} & ([j \varepsilon s / 2, \infty))-\mathbb{E}\left[\chi^{\mathrm{GOE}}([-j \varepsilon s / 2, \infty))\right] \\
& \leq k_{j}-\frac{2}{3 \pi}(j \varepsilon s / 2)^{3 / 2}-C_{2}(j \varepsilon s / 2) \\
& =\frac{(\varepsilon s)^{3 / 2}}{3 \pi \sqrt{2}}\left((j-1)^{3 / 2}-j^{3 / 2}\right)+C_{1}((j-1) \varepsilon s / 2)-C_{2}(j \varepsilon s / 2) \\
& \leq-M \sqrt{j}(\varepsilon s)^{3 / 2}+M^{\prime},
\end{aligned}
$$

where $M>0$ is a constant extracted from the fact that

$$
(j-1)^{3 / 2}-j^{3 / 2} \leq \underset{29}{\sqrt{j}}((j-1)-j)=-\sqrt{j} .
$$


It follows that

$$
\mathbb{P}\left(\mathrm{a}_{k_{j}} \leq p_{j}\right) \leq \mathbb{P}\left(\chi^{\mathrm{GOE}}\left(\left[p_{j}, \infty\right)\right)-\mathbb{E}\left[\chi^{\mathrm{GOE}}\left(\left[p_{j}, \infty\right)\right)\right] \leq-M \sqrt{j}(\varepsilon s)^{3 / 2}+M^{\prime}\right) .
$$

Now, for sufficiently large $S_{0}$, we have

$$
-M \sqrt{j}(\varepsilon s)^{3 / 2}+M^{\prime} \leq-\frac{M}{2} \sqrt{j}(\varepsilon s)^{3 / 2}
$$

for all $j \in\left\{1, \ldots,\left\lceil 2 \varepsilon^{-1}\right\rceil+1\right\}$ and for all $s \geq S_{0}$. Assuming Conjecture 1 , we may now apply equation (1.33) of Theorem 1.11: there exist $S_{0}(\varepsilon, \delta)$ and $K_{1}=K_{1}(\varepsilon, \delta)$ such that for all $s \geq S_{0}$,

$$
\mathbb{P}\left(\mathrm{a}_{k_{j}} \leq p_{j}\right) \leq \mathbb{P}\left(\chi^{\mathrm{GOE}}\left(\left[p_{j}, \infty\right)\right)-\mathbb{E}\left[\chi^{\mathrm{GOE}}\left(\left[p_{j}, \infty\right)\right)\right] \leq-\frac{M}{2} \sqrt{j}(\varepsilon s)^{3 / 2}\right) \leq \exp \left(K_{1} s^{3-\delta}\right) .
$$

This proves (7.21). Applying (1.32) instead of (1.33) above yields (7.19) (for all $s \geq S_{0}$, for some $\left.S_{0}:=S_{0}(\eta, \varepsilon, \delta)\right)$.

Towards showing (7.20) and (7.22), assume $s$ is large enough so that $\lambda_{\theta_{0}}<s$. We will now show that

$$
\bigcup_{k=1}^{\theta_{0}}\left\{D_{k} \geq \varepsilon s\right\} \subset \bigcup_{j=1}^{\left\lceil 2 \varepsilon^{-1}\right\rceil+1}\left\{\mathrm{a}_{k_{j}} \leq p_{j}\right\} .
$$

First, choose $1 \leq k \leq \theta_{0}$ and assume that $D_{k} \geq \varepsilon s$. There exists $1 \leq j \leq\left\lceil 2 \varepsilon^{-1}\right\rceil+1$ such that $-\lambda_{k} \in \mathcal{Q}_{j-1}$. The left boundary point of $\mathcal{Q}_{j-1}$ is $q_{j}$, and since $D_{k}=-\lambda_{k}-\mathrm{a}_{k} \geq \varepsilon s$, we have $\mathrm{a}_{k} \leq-\lambda_{k}-\varepsilon s$. Since $-\lambda_{k} \geq q_{j}$, by definition of $k_{j}$, we have $k_{j} \geq k$. Thus, $\mathrm{a}_{k} \geq \mathrm{a}_{k_{j}}$. It follows that

$$
\mathrm{a}_{k_{j}} \leq \mathrm{a}_{k} \leq-\lambda_{k}-\varepsilon s \leq-\lambda_{k_{j}}-\frac{\varepsilon s}{2}
$$

where the last inequality uses the fact that $\lambda_{k_{j}}, \lambda_{k} \in \mathcal{Q}_{j-1}$, and thus $0 \leq \lambda_{k_{j}}-\lambda_{k} \leq \varepsilon s / 2$. Hence, the distance between $a_{k_{j}}$ and $-\lambda_{k_{j}}$ is greater than or equal to $\varepsilon s / 2$, from which it follows that $\mathrm{a}_{k_{j}} \leq p_{j}$. This establishes (7.28).

Assuming Conjecture 1, we may combine (7.21) and (7.28) to obtain

$$
\mathbb{P}\left(\bigcup_{k=1}^{\theta_{0}}\left\{D_{k} \geq \varepsilon s\right\}\right) \leq \sum_{i=1}^{\left\lceil 2 \varepsilon^{-1}\right\rceil+1} \mathbb{P}\left(\mathrm{a}_{k_{i}} \leq p_{i}\right) \leq\left(\left\lceil 2 \varepsilon^{-1}\right\rceil+1\right) \exp \left(-K_{1} s^{3-\delta}\right) .
$$

For $S_{0}:=S_{0}(\varepsilon, \delta)$ sufficiently large, we can modify the constant $K_{1}:=K_{1}(\varepsilon, \delta)$ to absorb the constant $\left\lceil 2 \varepsilon^{-1}\right\rceil+1$. This establishes (7.22). On the other-hand, from (7.19) and (7.28), we obtain

$$
\mathbb{P}\left(\bigcup_{k=1}^{\theta_{0}}\left\{D_{k} \geq \varepsilon s\right\}\right) \leq \sum_{i=1}^{\left\lceil 2 \varepsilon^{-1}\right\rceil+1} \mathbb{P}\left(\mathrm{a}_{k_{i}} \leq p_{i}\right) \leq\left(\left\lceil 2 \varepsilon^{-1}\right\rceil+1\right) \exp \left(-\eta^{\prime} s^{3 / 2}\right),
$$

for any $\eta^{\prime}>0$. For any given $\eta>0$, we may choose $\eta^{\prime}$ sufficiently close to 0 and $S_{0}:=S_{0}(\eta, \varepsilon, \delta)$ sufficiently large such that

$$
\left(\left\lceil 2 \varepsilon^{-1}\right\rceil+1\right) \exp \left(-\eta^{\prime} s^{3 / 2}\right) \leq \exp \left(-\eta s^{3 / 2}\right) .
$$

Thus, we have (7.20). This completes the proof of Lemma 7.5.

7.2. Proof of the lower bound, equation (2.10). In this section we establish a lower bound on $\mathbb{E}\left[\prod_{k=1}^{\infty} I_{s}\left(\mathrm{a}_{k}\right)\right]$ by deriving an upper bound on $\sum_{k=1}^{\infty} J_{s}\left(\mathrm{a}_{k}\right)$. The result will lead us to (2.10) of Proposition 2.2, thus completing the proof of Theorem 1.4. We begin with an algebraic inequality from [CG20]. 
Lemma 7.6 ([CG20, Lemma 5.6]). For all $a>27$ and all $x \geq \sqrt{3 a}$, we have

$$
(a+x)^{2 / 3} \geq a^{2 / 3}+x^{1 / 3} .
$$

The following lemma gives the needed upper-bound on $\sum_{k=1}^{\infty} J_{s}\left(\mathrm{a}_{k}\right)$ when $\mathrm{a}_{1} \geq-s$ (see Claim 7.10).

Lemma 7.7. Fix $T_{0}>0$. There exist positive constants $S_{0}$ and $B:=B\left(T_{0}\right)$ such that for all $\varepsilon \in(0,1 / 3)$, for all $s \geq S_{0}$, and for all $T>T_{0}$, we have

$$
\sum_{k=1}^{\infty} J_{s}\left(\mathrm{a}_{k}\right) \leq \frac{1}{2} \mathcal{L}_{T, \varepsilon}\left(s+C_{\varepsilon}^{\mathrm{GOE}}\right)
$$

where

$$
\mathcal{L}_{T, \varepsilon}(x):=T^{1 / 3}\left(\frac{4 x^{5 / 2}}{15 \pi}(1+3 \varepsilon)+2 x-B\right)+\frac{x^{3 / 2}}{3(1-\varepsilon)^{3 / 2}}+\sqrt{\frac{3}{\pi}} \frac{x^{3 / 4}}{(1-\varepsilon)^{3 / 4}}+\frac{4}{T \pi(1-\varepsilon)^{3}} .
$$

Proof. Recall from (2.9) that $J_{s}(x)$ is a monotonically increasing function, and recall from (1.14) that $\mathrm{a}_{k} \leq-(1-\varepsilon) \lambda_{k}+C_{\varepsilon}^{\mathrm{GOE}}$, for all $k \in \mathbb{Z}_{>0}$. It follows that

$$
\sum_{k=1}^{\infty} J_{s}\left(\mathrm{a}_{k}\right) \leq \sum_{k=1}^{\infty} J_{s}\left(-(1-\varepsilon) \lambda_{k}+C_{\varepsilon}^{\mathrm{GOE}}\right)=(\widetilde{I})+(\widetilde{I I})+(\widetilde{I I I}),
$$

where $(\widetilde{I}),(\widetilde{I I})$, and $(\widetilde{I I I})$ equal the sum of $J_{s}\left(-(1-\varepsilon) \lambda_{k}+C_{\varepsilon}^{\mathrm{GOE}}\right)$ over all integers $k$ in the intervals $\left[1, \theta_{1}^{\prime}\right],\left(\theta_{1}^{\prime}, \theta_{2}^{\prime}\right)$, and $\left[\theta_{2}^{\prime}, \infty\right)$ respectively, and we define

$$
\begin{aligned}
& \theta_{1}^{\prime}:=\left\lceil 4 \sup _{n \in \mathbb{Z}_{>0}} n|\mathcal{R}(n)|\right\rceil, \text { and } \\
& \theta_{2}^{\prime}:=\left\lceil\frac{2\left(s+C_{\varepsilon}^{\mathrm{GOE}}\right)^{3 / 2}}{3 \pi(1-\varepsilon)^{3 / 2}}+\frac{1}{2}\right\rceil,
\end{aligned}
$$

where $\mathcal{R}(n)$ is defined as in Proposition 3.4. Since the $\lambda_{i}$ are strictly decreasing in $i$, we have

$$
J_{s}\left(-(1-\varepsilon) \lambda_{k}+C_{\varepsilon}^{\mathrm{GOE}}\right) \leq J_{s}\left(-(1-\varepsilon) \lambda_{1}+C_{\varepsilon}^{\mathrm{GOE}}\right),
$$

for all $k \geq 1$. Using this and the inequality $\log (1+\exp (a)) \leq a+\pi / 2$ for any $a>0$, we obtain

$$
(\widetilde{I}) \leq \theta_{1}^{\prime} J_{s}\left(-(1-\varepsilon) \lambda_{1}+C_{\varepsilon}^{\mathrm{GOE}}\right) \leq \frac{1}{2}\left(\theta_{1}^{\prime} T^{1 / 3}\left(s-(1-\varepsilon) \lambda_{1}+C_{\varepsilon}^{\mathrm{GOE}}\right)+\frac{\pi \theta_{1}^{\prime}}{2}\right) .
$$

Terms $(\widetilde{I I})$ and $(\widetilde{I I I})$ are bounded in the following two claims.

Claim 7.8. For all $s>0$, we have

$2(\widetilde{I I}) \leq T^{1 / 3}\left(\frac{4\left(s+C_{\varepsilon}^{\mathrm{GOE}}\right)^{5 / 2}}{15 \pi}(1+3 \varepsilon)+\left(2-\theta_{1}^{\prime}\right)\left(s+C_{\varepsilon}^{\mathrm{GOE}}\right)-\frac{3}{5}\left(\frac{3 \pi}{2}\right)^{2 / 3}\left(\theta_{1}^{\prime}\right)^{5 / 3}\right)+\frac{\pi\left(\theta_{2}^{\prime}-\theta_{1}^{\prime}\right)}{2}$.

Proof of Claim 7.8. Recall the constant $\mathcal{K}$, defined in (7.4). It follows that for $k \in\left(\theta_{1}^{\prime}, \infty\right)$, we have

$$
|\mathcal{R}(k)| \leq \frac{\mathcal{K}}{k} \leq \frac{\mathcal{K}}{\theta_{1}^{\prime}} \leq 1 / 4
$$

Combining this with Proposition 3.4, we find

$$
\lambda_{k} \geq\left(\frac{3 \pi\left(k-\frac{1}{4}-|\mathcal{R}(k)|\right)}{2}\right)_{31}^{2 / 3} \geq\left(\frac{3 \pi\left(k-\frac{1}{2}\right)}{2}\right)^{2 / 3}
$$


Using this, the inequality $\log (1+\exp (a)) \leq a+\pi / 2$ for any $a>0$, and the monotonicity of $J_{s}(\cdot)$, we obtain

$$
(\widetilde{I I}) \leq \frac{1}{2} \sum_{k=\theta_{1}^{\prime}+1}^{\theta_{2}^{\prime}-1}\left(T^{1 / 3} f_{s}(k)+\frac{\pi}{2}\right)
$$

where

$$
f_{s}(z):=s+C_{\varepsilon}^{\mathrm{GOE}}-(1-\varepsilon)\left(\frac{3 \pi\left(z-\frac{1}{2}\right)}{2}\right)^{2 / 3} .
$$

Since $f_{s}(z)$ is a monotonically decreasing function of $z$, we may bound the sum in (7.37) with an integral:

$$
\frac{1}{2} \sum_{k=\theta_{1}^{\prime}+1}^{\theta_{2}^{\prime}-1}\left(T^{1 / 3} f_{s}(k)+\frac{\pi}{2}\right) \leq \frac{1}{2}\left(T^{1 / 3} \int_{\theta_{1}^{\prime}}^{\theta_{2}^{\prime}} f_{s}(z) d z+\frac{\pi\left(\theta_{2}^{\prime}-\theta_{1}^{\prime}\right)}{2}\right) .
$$

We now compute

$$
\begin{aligned}
\int_{\frac{1}{2}}^{\theta_{2}^{\prime}} f_{s}(z) d z & =\left(s+C_{\varepsilon}^{\mathrm{GOE}}\right)\left(\theta_{2}^{\prime}-\frac{1}{2}\right)-\frac{3(1-\varepsilon)}{5}\left(\frac{3 \pi}{2}\right)^{2 / 3}\left(\theta_{2}^{\prime}-\frac{1}{2}\right)^{5 / 3} \\
& \leq\left(s+C_{\varepsilon}^{\mathrm{GOE}}\right)\left(\frac{2\left(s+C_{\varepsilon}^{\mathrm{GOE}}\right)^{3 / 2}}{3 \pi(1-\varepsilon)^{3 / 2}}+\frac{3}{2}\right)-\frac{3(1-\varepsilon)}{5}\left(\frac{3 \pi}{2}\right)^{2 / 3}\left(\frac{2\left(s+C_{\varepsilon}^{\mathrm{GOE}}\right)^{3 / 2}}{3 \pi(1-\varepsilon)^{3 / 2}}\right)^{5 / 3} \\
& =\frac{4\left(s+C_{\varepsilon}^{\mathrm{GOE}}\right)^{5 / 2}}{15(1-\varepsilon)^{3 / 2}}+\frac{3}{2}\left(s+C_{\varepsilon}^{\mathrm{GOE}}\right) \\
& \leq \frac{4\left(s+C_{\varepsilon}^{\mathrm{GOE}}\right)^{5 / 2}}{15}(1+3 \varepsilon)+\frac{3}{2}\left(s+C_{\varepsilon}^{\mathrm{GOE}}\right)
\end{aligned}
$$

and

$$
\begin{aligned}
\int_{\frac{1}{2}}^{\theta_{1}^{\prime}} f_{s}(z) d z & \geq\left(s+C_{\varepsilon}^{\mathrm{GOE}}\right)\left(\theta_{1}^{\prime}-\frac{1}{2}\right)-\int_{\frac{1}{2}}^{\theta_{1}^{\prime}}\left(\frac{3 \pi\left(z-\frac{1}{2}\right)}{2}\right)^{2 / 3} d z \\
& =\left(s+C_{\varepsilon}^{\mathrm{GOE}}\right)\left(\theta_{1}^{\prime}-\frac{1}{2}\right)-\frac{3}{5}\left(\frac{3 \pi}{2}\right)^{2 / 3}\left(\theta_{1}^{\prime}\right)^{5 / 3} .
\end{aligned}
$$

Substituting the bounds from (7.39) and (7.40) into (7.38) yields the upper bound on $(\widetilde{I I})$ in $(7.35)$. This completes the proof of Claim 7.8.

Claim 7.9. There exists a positive constant $S_{0}>0$ such that for all $s \geq S_{0}$, we have

$$
(\widetilde{I I I}) \leq \frac{1}{2}\left(\sqrt{\frac{3}{\pi}} \frac{\left(s+C_{\varepsilon}^{\mathrm{GOE}}\right)^{3 / 4}}{(1-\varepsilon)^{3 / 4}}+\frac{4}{T \pi(1-\varepsilon)^{3}}\right) .
$$

Proof of Claim 7.9. Using the inequality $\log (1+z) \leq z$ for all $z \geq 0$, we obtain

$$
J_{s}\left(-(1-\varepsilon) \lambda_{k}+C_{\varepsilon}^{\mathrm{GOE}}\right) \leq \frac{1}{2} \exp \left(T^{1 / 3}\left(s-(1-\varepsilon) \lambda_{k}+C_{\varepsilon}^{\mathrm{GOE}}\right)\right) .
$$

Recalling the lower bound on $\lambda_{k}$ from (7.36) and the definition of $f_{s}(z)$ from (7.37), we find

$$
(\widetilde{I I I}) \leq \frac{1}{2} \sum_{k=\theta_{2}^{\prime}}^{\infty} \exp \left(T^{1 / 3} f_{s}(k)\right)
$$


For all $k \geq \theta_{2}^{\prime}$, we have

$$
s+C_{\varepsilon}^{\mathrm{GOE}}<(1-\varepsilon)\left(\frac{3 \pi\left(\theta_{2}^{\prime}-\frac{1}{2}\right)}{2}\right)^{2 / 3} .
$$

Since $f_{s}(z)$ is a monotonically decreasing function, we have $f_{s}(k) \leq f_{s}\left(\theta_{2}^{\prime}\right)<0$ for all $k \geq \theta_{2}^{\prime}$. Thus, for all $k>\theta_{2}^{\prime}+\sqrt{3 \theta_{2}^{\prime}}, S_{0}$ sufficiently large, and for all $s \geq S_{0}$, we may write

$$
f_{s}(k)<(1-\varepsilon)\left(\left(\frac{3 \pi\left(\theta_{2}^{\prime}-\frac{1}{2}\right)}{2}\right)^{2 / 3}-\left(\frac{3 \pi\left(k-\frac{1}{2}\right)}{2}\right)^{2 / 3}\right) \leq-(1-\varepsilon)\left(\frac{3 \pi\left(k-\theta_{2}^{\prime}\right)}{2}\right)^{1 / 3}
$$

where the last inequality uses (7.31) with

$$
a:=\frac{3 \pi}{2}\left(\theta_{2}^{\prime}-\frac{1}{2}\right), \quad x:=\frac{3 \pi}{2}\left(k-\theta_{2}^{\prime}\right)
$$

$\left(S_{0}\right.$ need only be large enough so that $a$ and $x$ as above satisfy the conditions of Lemma 7.6 for all $\left.s \geq S_{0}\right)$. It follows from $(7.44)$ and $f_{s}(k)<0$ that

$$
\exp \left(T^{1 / 3} f_{s}(k)\right) \leq\left\{\begin{array}{ll}
1, & \text { for } k \in\left[\theta_{2}^{\prime}, \theta_{2}^{\prime}+\sqrt{3 \theta_{2}^{\prime}}\right) \\
\exp \left(-(1-\varepsilon)\left(\frac{3 \pi\left(k-\theta_{2}^{\prime}\right)}{2}\right)^{1 / 3}\right), & \text { for } k \in\left[\theta_{2}^{\prime}+\sqrt{3 \theta^{\prime}}, \infty\right)
\end{array},\right.
$$

for $S_{0}$ sufficiently large and for all $s \geq S_{0}$. From (7.43) and the above, we find that for $S_{0}$ sufficiently large and all $s \geq S_{0}$,

$$
\begin{aligned}
2(\widetilde{I I I}) & \leq \sum_{k \in\left[\theta_{2}^{\prime}, \theta_{2}^{\prime}+\sqrt{3 \theta_{2}^{\prime}}\right)} \exp \left(T^{1 / 3} f_{s}(k)\right)+\sum_{k \geq \theta_{2}^{\prime}+\sqrt{3 \theta_{2}^{\prime}}} \exp \left(T^{1 / 3} f_{s}(k)\right) \\
& \leq 1+\sqrt{3 \theta_{2}^{\prime}}+\sum_{k=\theta_{2}^{\prime}+\sqrt{3 \theta^{\prime}}}^{\infty} \exp \left(-(1-\varepsilon)\left(\frac{3 \pi\left(k-\theta_{2}^{\prime}\right)}{2}\right)^{1 / 3}\right) \\
& \leq 1+\sqrt{3 \theta_{2}^{\prime}}+\int_{0}^{\infty} \exp \left(-(1-\varepsilon) T^{1 / 3}\left(\frac{3 \pi z}{2}\right)^{1 / 3}\right) d z \\
& =1+\sqrt{3 \theta_{2}^{\prime}}+\frac{4}{T \pi(1-\varepsilon)^{3}} \\
& \leq \sqrt{\frac{3}{\pi}} \frac{\left(s+C_{\varepsilon}^{\mathrm{GOE}}\right)^{3 / 4}}{(1-\varepsilon)^{3 / 4}}+\frac{4}{T \pi(1-\varepsilon)^{3}} .
\end{aligned}
$$

This completes the proof of (7.41) of Claim 7.9.

We now return to the proof of Lemma 7.7. Define the bounded, positive constant

$$
B^{\prime}:=\frac{3}{5}\left(\frac{3 \pi}{2}\right)^{2 / 3}\left(\theta_{1}^{\prime}\right)^{5 / 3}+(1-\varepsilon) \theta_{1}^{\prime} \lambda_{1} .
$$

Then substituting the bounds given by (7.34), (7.35), and (7.41) into (7.33) yields

$$
\begin{aligned}
2 \sum_{k=1}^{\infty} J_{s}\left(\mathrm{a}_{k}\right) & \leq T^{1 / 3}\left(\frac{4\left(s+C_{\varepsilon}^{\mathrm{GOE}}\right)^{5 / 2}}{15 \pi}(1+3 \varepsilon)+2\left(s+C_{\varepsilon}^{\mathrm{GOE}}\right)-B^{\prime}\right)+\frac{\pi \theta_{2}^{\prime}}{2} \\
& +\sqrt{\frac{3}{\pi}} \frac{\left(s+C_{\varepsilon}^{\mathrm{GOE}}\right)^{3 / 4}}{(1-\varepsilon)^{3 / 4}}+\frac{4}{T \pi(1-\varepsilon)^{3}} .
\end{aligned}
$$


Now,

$$
\frac{\pi \theta_{2}^{\prime}}{2} \leq \frac{\pi}{2}\left(\frac{2}{3 \pi} \frac{\left(s+C_{\varepsilon}^{\mathrm{GOE}}\right)^{3 / 2}}{(1-\varepsilon)^{3 / 2}}+\frac{3}{2}\right)=\frac{\left(s+C_{\varepsilon}^{\mathrm{GOE}}\right)^{3 / 2}}{3(1-\varepsilon)^{3 / 2}}+\frac{3 \pi}{4} .
$$

Taking $B:=B^{\prime}-\frac{3 \pi}{4 T_{0}^{1 / 3}}$ yields $(7.32)$.

Proof of (2.10) of Proposition 2.2. In what follows, we fix $\varepsilon \in(0,1 / 3), \delta \in(0,1 / 4)$, and $T_{0}>0$. We begin with two claims.

Claim 7.10. There exist $\kappa:=\kappa(\varepsilon, \delta)>0$ and $S_{0}=S_{0}\left(\varepsilon, \delta, T_{0}\right)>0$ such that, for all $s \geq S_{0}$ and $T>T_{0}$,

$$
\mathbb{E}_{\mathrm{GOE}}\left[\mathbb{1}\left(\mathrm{a}_{1} \geq-s\right) \prod_{k=1}^{\infty} I\left(\mathrm{a}_{k}\right)\right] \geq\left(1-2 \kappa \exp \left(-\kappa s^{1-2 \delta}\right)\right) \exp \left(-\frac{2 T^{1 / 3} s^{5 / 2}}{15 \pi}(1+9 \varepsilon)\right) .
$$

Proof of Claim 7.10. Negating both sides of (7.32) and then exponentiating yields

$$
\prod_{k=1}^{\infty} I\left(\mathrm{a}_{k}\right) \geq \exp \left(-\frac{1}{2} \mathcal{L}_{T, \varepsilon}\left(s+C_{\varepsilon}^{\mathrm{GOE}}\right)\right) .
$$

Since $\mathcal{L}_{T, \varepsilon}(x)$ is monotonically increasing, we may bound

$$
\mathbb{E}_{\mathrm{GOE}}\left[\mathbb{1}\left(\mathrm{a}_{1} \geq-s\right) \prod_{k=1}^{\infty} I\left(\mathrm{a}_{k}\right)\right] \geq \mathbb{P}\left(\mathrm{a}_{1} \geq-s, C_{\varepsilon}^{\mathrm{GOE}}<s^{1-\delta}\right) \exp \left(-\frac{1}{2} \mathcal{L}_{T, \varepsilon}\left(s+s^{1-\delta}\right)\right) .
$$

Take $S_{0}>0$ large enough so that for all $s \geq S_{0}$,

$$
\mathcal{L}_{T, \varepsilon}\left(s+s^{1-\delta}\right) \leq T^{1 / 3} \frac{4 s^{5 / 2}}{15 \pi}(1+9 \varepsilon) .
$$

From Theorem 1.5, there exist $\kappa:=\kappa(\varepsilon, \delta)$ and a (potentially larger) $S_{0}$ such that. for all $s \geq S_{0}$,

$$
\mathbb{P}\left(C_{\varepsilon}^{\mathrm{GOE}}<s^{1-\delta}\right)>1-\kappa \exp \left(-\kappa s^{1-2 \delta}\right) .
$$

Furthermore, for large enough $S_{0}$, we find from (7.1) that for all $s \geq S_{0}$,

$$
\mathbb{P}\left(\mathrm{a}_{1}<-s\right) \leq \exp \left(-\frac{1}{24} s^{3}(1+o(1))\right) \leq \kappa \exp \left(-\kappa s^{1-2 \delta}\right) .
$$

Thus, for large enough $S_{0}$, we have

$$
\mathbb{P}\left(\mathrm{a}_{1} \geq-s, C_{\varepsilon}^{\mathrm{GOE}}<s^{1-\delta}\right) \geq \mathbb{P}\left(\mathrm{a}_{1} \geq-s\right)+\mathbb{P}\left(C_{\varepsilon}^{\mathrm{GOE}}<s^{1-\delta}\right)-1 \geq 1-2 \kappa \exp \left(-\kappa s^{1-2 \delta}\right) .
$$

Plugging this and (7.52) into (7.51) yields equation (7.50) of Claim 7.10.

Claim 7.11. There exist constants $K_{2}:=K_{2}\left(T_{0}\right)>0$ and $S_{0}:=S_{0}\left(\varepsilon, \delta, T_{0}\right)>0$ such that for all $s \geq S_{0}$, we have

$$
\mathbb{E}_{\mathrm{GOE}}\left[\mathbb{1}\left(\mathrm{a}_{1}<-s\right) \prod_{k=1}^{\infty} I\left(\mathrm{a}_{k}\right)\right] \geq \exp \left(-K_{2} s^{3}\right) .
$$

Proof of Claim 7.11. Define the parameter $L:=\frac{3}{1-\delta}$, and note that $L \in(3,4]$. Let $\mathfrak{J}$ denote the interval $\left[-s^{L},-s\right)$. We seek an upper bound first on $\sum_{\mathrm{a}_{k} \in \mathfrak{J}} J_{s}\left(\mathrm{a}_{k}\right)$ and then on $\sum_{\mathrm{a}_{k}<-s^{L}} J_{s}\left(\mathrm{a}_{k}\right)$. Since $J_{s}(\cdot)$ is monotonically increasing, we obtain the following upper bound by replacing all the $\mathrm{a}_{k}$ 's inside the interval $\mathfrak{J}$ by the right endpoint $s$ of the interval:

$$
\sum_{\mathrm{a}_{k} \in \mathfrak{J}} J_{s}\left(\mathrm{a}_{k}\right) \leq \chi^{\mathrm{GOE}}(\mathfrak{J}) J_{s}(-s)=\frac{1}{2} \chi^{\mathrm{GOE}}\left(\mathfrak{J}_{\ell}\right) \log 2 .
$$


Next, using Theorem 1.12, there exists $\mathcal{C}:=\mathcal{C}(\varepsilon, \delta)$ and $S_{0}:=S_{0}(\varepsilon)$ such that for all $s \geq S_{0}$, we have

$$
\chi^{\mathrm{GOE}}(\mathfrak{J}) \leq \mathbb{E}\left[\chi^{\mathrm{GOE}}(\mathfrak{J})\right]+\varepsilon s^{3 L / 2}
$$

holds with probability greater than or equal to $1-\exp \left(-\mathcal{C} s^{3}\right)$. In what follows, we will write $C$ to denote a positive constant independent of $\varepsilon \in(0,1 / 3)$ and $\delta \in(0,1 / 4)$ (but may depend on $T_{0}$ ) whose value may change from line to line. Then from Theorem 1.6, we have for large enough $s$

$$
\mathbb{E}\left[\chi^{\mathrm{GOE}}(\mathfrak{J})\right]=\frac{2}{3 \pi}\left(s^{3 L / 2}-s^{3 / 2}\right)+\mathfrak{D}_{1}\left(s^{L}\right)-\mathfrak{D}_{1}(s) \leq C s^{3 L / 2} .
$$

Substituting this into (7.55), we may deduce that

$$
\sum_{\mathrm{a}_{k} \in \mathfrak{J}} J\left(\mathrm{a}_{k}\right) \leq C s^{3 L / 2}
$$

holds with probability greater than or equal to $1-\exp \left(-\mathcal{C} s^{3}\right)$.

It remains to bound the sum $\sum_{\mathrm{a}_{k}<-s^{L}} J_{s}\left(\mathrm{a}_{k}\right)$, which we now decompose into two sums:

$$
\begin{gathered}
\sum_{\mathrm{a}_{k}<-s^{L}} J_{s}\left(\mathrm{a}_{k}\right)=(\mathbf{A})+(\mathbf{B}), \text { where } \\
\sum_{\left\{k: \mathrm{a}_{k}<-s^{L}, \lambda_{k} \leq s^{L}\right\}} J_{s}\left(\mathrm{a}_{k}\right), \quad(\mathbf{B}):=\sum_{\left\{k: \mathrm{a}_{k}<-s^{L}, \lambda_{k}>s^{L}\right\}} J_{s}\left(\mathrm{a}_{k}\right) .
\end{gathered}
$$

Using the bound $\log (1+a) \leq a$ for all $a \geq 0$ gives

$$
J_{s}\left(\mathrm{a}_{k}\right) \leq \frac{1}{2} \exp \left(T^{1 / 3}\left(s-s^{L}\right)\right) \leq \frac{1}{2} \exp \left(-(1-\varepsilon) T^{1 / 3} s^{3}\right)
$$

for $\mathrm{a}_{k} \leq-s^{L}, S_{0}:=S_{0}(\varepsilon, \delta)$ large enough, and all $s \geq S_{0}$. Corollary 3.5 shows

$$
\#\left\{k: \lambda_{k} \leq s^{L}\right\}=\frac{2}{3 \pi} s^{3 L / 2}+C_{1}\left(s^{L}\right) \leq C s^{3 L / 2} .
$$

Thus, for large enough $S_{0}$, we have

$$
\left.(\mathbf{A}) \leq \frac{1}{2} C s^{3 L / 2} \exp \left(-(1-\varepsilon) T^{1 / 3} s^{3}\right)\right) \leq s^{3} .
$$

We now bound (B). From monotonicity and (1.14), we have $J_{s}\left(\mathrm{a}_{k}\right) \leq J_{s}\left(-(1-\varepsilon) \lambda_{k}+C_{\varepsilon}^{\mathrm{GOE}}\right)$, where $C_{\varepsilon}^{\mathrm{GOE}}$ is as defined in Theorem 1.5. We now employ Theorem 1.5, taking $\tilde{s}$ and $\tilde{\delta}$ as our variables instead of the $s$ and $\delta$ in the notation of the theorem to avoid confusion (though we take the $\varepsilon$ in the statement of Theorem 1.5 to be the same as our $\varepsilon$ here). With $\tilde{s}:=s^{3+\frac{\delta}{2}}$ and $\tilde{\delta}:=\frac{\delta}{2(3+\delta / 2)}$, Theorem 1.5 implies that there exist $\kappa:=\kappa(\varepsilon, \delta)>0$ and $S_{0}:=S_{0}(\varepsilon, \delta)>0$ such that for all $s \geq S_{0}$, we have

$$
\mathbb{P}\left(C_{\varepsilon}^{\mathrm{GOE}}<s^{3+\frac{\delta}{2}}\right) \geq 1-\kappa \exp \left(-\kappa s^{3}\right) .
$$

Now, for large enough $S_{0}$, we have $s+s^{3+\frac{\delta}{2}} \leq(1-\varepsilon) s^{L}$. Since $s^{L}<\lambda_{k}$ in $(\mathbf{B})$, we have for large enough $S_{0}$

$$
\mathbb{P}\left((\mathbf{B}) \leq \sum_{\lambda_{k}>s^{L}} J_{s}\left((1-\varepsilon)\left(s^{L}-\lambda_{k}\right)-s\right)\right) \geq 1-\kappa \exp \left(-\kappa s^{3}\right) .
$$

The bounds in (7.60), (7.61), and (7.67) of Claim 7.12 (given below), as well as the bound $3 L / 4 \leq 3$, we find that for $S_{0}$ large enough,

$$
\mathbb{P}\left((\mathbf{A})+(\mathbf{B}) \leq C s^{3}\right) \geq 1-\kappa \exp \left(-\kappa s^{3}\right)
$$


Combining this bound with the bound in (7.57) yields

$$
\mathbb{P}(\mathcal{A}) \geq 1-\exp \left(-\mathcal{C} s^{3}\right)-\kappa \exp \left(-\kappa s^{3}\right)
$$

where $\mathcal{A}:=\left\{\sum_{k=1}^{\infty} J_{s}\left(\mathrm{a}_{k}\right) \leq C s^{3}\right\}$. We then obtain

$$
\mathbb{E}_{\mathrm{GOE}}\left[\mathbb{1}\left(\mathrm{a}_{1}<-s\right) \prod_{k=1}^{\infty} I\left(\mathrm{a}_{k}\right)\right] \geq \mathbb{P}\left(\left\{\mathrm{a}_{1}<-s\right\} \cap \mathcal{A}\right) \exp \left(-C s^{3}\right) .
$$

We finally estimate, for a constant $K_{2}>0$ and for large enough $S_{0}$,

$$
\begin{aligned}
\mathbb{P}\left(\left\{\mathrm{a}_{1} \leq-s\right\} \cap \mathcal{A}\right) & \geq \mathbb{P}\left(\mathrm{a}_{1} \leq-s\right)+\mathbb{P}(\mathcal{A})-1 \\
& \geq \exp \left(-s^{3}\right)-\exp \left(-\mathcal{C} s^{3}\right)-\kappa \exp \left(-\kappa s^{3}\right) \\
& \geq \exp \left(-C^{\prime} s^{3}\right),
\end{aligned}
$$

where the first inequality uses $\mathbb{P}(A \cap B) \geq \mathbb{P}(A)+\mathbb{P}(B)-1$ for any events $A$ and $B$, and the second inequality uses (7.1) and the lower bound in (7.63). Substituting (7.65) into (7.64) yields (7.53). This concludes the proof of Claim 7.11.

We may now complete the proof of (2.10) of Proposition 2.2 by substituting (7.50) and (7.53) into

$$
\mathbb{E}_{\mathrm{GOE}}\left[\prod_{k=1}^{\infty} I\left(\mathrm{a}_{k}\right)\right]=\mathbb{E}_{\mathrm{GOE}}\left[\mathbb{1}\left(\mathrm{a}_{1} \geq-s\right) \prod_{k=1}^{\infty} I\left(\mathrm{a}_{k}\right)\right]+\mathbb{E}_{\mathrm{GOE}}\left[\mathbb{1}\left(\mathrm{a}_{1}<-s\right) \prod_{k=1}^{\infty} I\left(\mathrm{a}_{k}\right)\right] .
$$

Claim 7.12. Fix $\varepsilon \in(0,1 / 3), \delta \in(0,1 / 4)$ and $T_{0}>0$. There exists a positive constant $S_{0}:=$ $S_{0}(\varepsilon, \delta)$ such that for all $s \geq S_{0}$, we have

$$
\sum_{\lambda_{k}>s^{L}} J_{s}\left((1-\varepsilon)\left(s^{L}-\lambda_{k}\right)-s\right) \leq C s^{3 L / 4} .
$$

Proof. For sufficiently large $s,(3.15)$ implies that

$$
\left\{k: \lambda_{k}>s^{L}\right\} \subseteq\left\{k: k>\frac{2}{3 \pi}\left(s^{L}\right)^{3 / 2}-\frac{3}{4}\right\} .
$$

This gives

$$
\sum_{\lambda_{k}>s^{L}} J_{s}\left((1-\varepsilon)\left(s^{L}-\lambda_{k}\right)-s\right) \leq \sum_{k>\frac{2}{3 \pi} s^{3 L / 2}-\frac{3}{4}} J_{s}\left((1-\varepsilon)\left(s^{L}-\lambda_{k}\right)-s\right) .
$$

To simplify the calculations that follow, we denote $\theta_{0}:=\frac{2}{3 \pi} s^{3 L / 2}-\frac{3}{4}$ and $\theta_{0}^{\prime}:=\theta_{0}+\sqrt{\frac{2}{\pi}} s^{3 L / 4}$. Note that for $\lambda_{k}>\theta_{0}$, we have $(1-\varepsilon)\left(s^{L}-\lambda_{k}\right)-s<0$ for sufficiently large $S_{0}$. We then use the fact that, for $x \leq-s$, we have $J_{s}(x) \leq \frac{1}{2} \log 2$. This is the bound we take on $J_{s}(\cdot)$ for $k \in\left[\theta_{0}, \theta_{0}^{\prime}\right]$.

For $k>\theta_{0}^{\prime}$, we recall the inequality $\log (1+z) \leq z$ for $z \geq 0$, which gives

$$
J_{s}\left((1-\varepsilon)\left(s^{L}-\lambda_{k}\right)-s\right) \leq \frac{1}{2} \exp \left((1-\varepsilon) T^{1 / 3}\left(s^{L}-\lambda_{k}\right)\right) .
$$

Define $\bar{k}:=k-\frac{1}{4}+\mathcal{R}(n)$ and $k^{\prime}:=k-\theta_{0}$, and note that $\bar{k}>\theta_{0}$ for $k>\theta_{0}^{\prime}$. Then Taylor's theorem yields

$$
s^{L}-\lambda_{k}=\left(\frac{3 \pi}{2}\left(\theta_{0}+\frac{3}{4}\right)\right)^{2 / 3}-\left(\frac{3 \pi}{2} \bar{k}\right)^{2 / 3} \leq-C\left(k^{\prime}\right)^{2 / 3}
$$


Now, substituting the bound given in (7.71) into (7.70) yields

$$
J_{s}\left((1-\varepsilon)\left(s^{L}-\lambda_{k}\right)-s\right) \leq\left\{\begin{array}{ll}
\frac{1}{2} \log 2 & k \in\left[\theta_{0}, \theta_{0}^{\prime}\right] \cap \mathbb{Z} \\
\frac{1}{2} \exp \left(-C(1-\varepsilon) T^{1 / 3}\left(k^{\prime}\right)^{2 / 3}\right) & k \in\left(\theta_{0}^{\prime}, \infty\right) \cap \mathbb{Z}
\end{array} .\right.
$$

From this bound, we have

$$
\begin{aligned}
\sum_{\lambda_{k}>s^{L}} J_{s}\left((1-\varepsilon)\left(s^{L}-\lambda_{k}\right)-s\right) & \leq \frac{1}{2}\left(\theta_{0}^{\prime}-\theta_{0}\right) \log 2+\frac{1}{2} \sum_{k^{\prime}>\theta_{0}^{\prime}-\theta_{0}} \exp \left(-C(1-\varepsilon) T^{1 / 3}\left(k^{\prime}\right)^{2 / 3}\right) \\
& \leq \frac{1}{\sqrt{2 \pi}} s^{3 L / 4} \log 2+\frac{C}{(1-\varepsilon) T^{1 / 3}} \\
& \leq C s^{3 L / 4},
\end{aligned}
$$

where the second-to-last inequality follows by bounding the sum with an integral. This gives the claim. 


\section{REFERENCES}

[ACQ11] G. Amir, I. Corwin, and J. Quastel. Probability distribution of the free energy of the continuum directed random polymer in $1+1$ dimensions. Commun. Pure Appl. Math., 64, 2011.

[AGZ10] G. W. Anderson, A. Guionnet, and O. Zeitouni. An Introduction to Random Matrices, volume 118. Cambridge Studies in Advanced Mathematics, 2010.

[AS77a] M. J. Ablowitz and H. Segur. Asymptotic solutions of the Korteweg-deVries equation. Studies in Appl. Math., 57:13-44, 1977.

[AS77b] M. J. Ablowitz and H. Segur. Exact linearization of a Painlevé transcendent. Phys. Rev. Lett., 38:11031106, 1977.

[BB18] T. Bothner and R. Buckingham. Large deformations of the Tracy-Widom distribution I. Non-oscillatory asymptotics. Commun. Math. Phys., 359:223-263, 2018.

[BBC16] A. Borodin, A. Bufetov, and I. Corwin. Directed random polymers via nested contour integrals. Ann. Physics, 368:191-247, 2016.

[BBC20] G. Barraquand, A. Borodin, and I. Corwin. Half-space Macdonald processes. Forum of Mathematics, Pi, 8:E11, 2020.

[BBCS18] J. Baik, G. Barraquand, I. Corwin, and T. Suidan. Pfaffian Schur processes and last passage percolation in a half-quadrant. Ann. Probab., 46(6):3015-3089, 2018.

[BBCW18] G. Barraquand, A. Borodin, I. Corwin, and M. Wheeler. Stochastic six-vertex model in a half-quadrant and half-line open ASEP. Duke Math. J., 167(13):2457-2529, 2018.

[BBD08] J. Baik, R. Buckingham, and J. DiFranco. Asymptotics of Tracy-Widom distributions and the total integral of a Painlevé II function. Commun. Math. Phys., 280, 2008.

[BdCP09] O. Bohigas, J. X. de Carvalho, and M. P. Pato. Deformations of the tracy-widom distribution. Phys. Rev. E, 79:031117, Mar 2009.

[BFO20] Dan Betea, Patrik L. Ferrari, and Alessandra Occelli. Stationary half-space last passage percolation. Communications in Mathematical Physics, 377(1):421-467, 2020.

[BG16] A. Borodin and V. Gorin. Moments match between the KPZ equation and the Airy point process. SIGMA Symmetry Integrability Geom. Methods Appl., 12(102), 2016.

[BKLD20] G. Barraquand, A. Krajenbrink, and P. Le Doussal. Half-space stationary kardar-parisi-zhang equation. Journal of Statistical Physics, 181(4):1149-1203, Aug 2020.

[Bot] T. Bothner. personal communication.

[Bot17] T. Bothner. Transition asymptotics for the Painlevé II transcendent. Duke Math. J., 166(2):205-324, 2017.

[BR01] J. Baik and E. Rains. The asymptotics of monotone subsequences of involutions. Duke Math. J., 109(2):205-281, 2001.

[CG20] I. Corwin and P. Ghosal. Lower tail of the KPZ equation. Duke Math. J., 169(7):1329-1395, 2020.

$\left[\mathrm{CGK}^{+} 18\right]$ I. Corwin, P. Ghosal, A. Krajenbrink, P. Le Doussal, and L.-C. Tsai. Coulomb-gas electrostatics controls large fluctuations of the Kardar-Parisi-Zhang equation. Phys. Rev. Lett, 121(6):060201, 2018.

[CLDR10] P. Calabrese, P. Le Doussal, and A. Rosso. Free-energy distribution of the directed polymer at high temperature. Euro. Phys. Lett., 90(2), 2010.

[Cor12] I. Corwin. The Kardar-Parisi-Zhang equation and universality class. Random Matrices Theory Appl., 1(1), 2012.

[Cor14] I. Corwin. Macdonald processes, quantum integrable systems and the kardar-parisi-zhang universality class. arXiv:1403.6877, 2014.

[CS18] I. Corwin and H. Shen. Open ASEP in the weakly asymmetric regime. Commun. Pure Appl. Math., 71(10):2065-2128, 2018.

[Dot10] V. Dotsenko. Bethe ansatz derivation of the Tracy-Widom distribution for one-dimensional directed polymers. Euro. Phys. Lett., 90(2), 2010.

[ES07] A. Edelman and B. D. Sutton. From random matrices to stochastic operators. J. Stat. Phys., 127(6):11211165, 2007.

[Fer10] P. L. Ferrari. From interacting particle systems to random matrices. Journal of Statistical Mechanics: Theory and Experiment, 2010(10):P10016, Oct 2010.

[For10] P. J. Forrester. Log-Gases and Random Matrices. London Mathematical Society monographs ; new ser. no. 34. Princeton University Press, 2010.

[GLD12] T. Gueudré and P. Le Doussal. Directed polymer near a hard wall and KPZ equation in the half-space. Europhys. Lett., 100(2), 2012.

[HM80] S. P. Hastings and J. B. McLeod. A boundary value problem associated with the second Painlevé transcendent and the Korteweg-deVries equation. Arch. Rational Mech. Anal., 73(1):31-51, 1980. 
[IS04] T. Imamura and T. Sasamoto. Fluctuations of the one-dimensional polynuclear growth model in halfspace. J. Stat. Phys, 115(3):749-803, 2004.

[Kar14] V. Kargin. On Pfaffian random point fields. J. Stat. Phys, 154(3):681-704, 2014.

[KLD18a] A. Krajenbrink and P. Le Doussal. Large fluctuations of the KPZ equation in a half-space. SciPost Phys, 5:32, 2018.

[KLD18b] A. Krajenbrink and P. Le Doussal. Simple derivation of the $(-\lambda h)^{5 / 2}$ tail for the 1D KPZ equation. J. Stat. Mech., 2018(6):063210, 2018.

[KLD20] A. Krajenbrink and P. Le Doussal. Replica Bethe Ansatz solution to the Kardar-Parisi-Zhang equation on the half-line. SciPost Phys., 8:35, 2020.

[KLDP18] A. Krajenbrink, P. Le Doussal, and S. Prolhac. Systematic time expansion for the Kardar-Parisi-Zhang equation, linear statistics of the GUE at the edge and trapped fermions. Nuclear Physics B, 936:239-305, 2018.

[Lin20] Y. Lin. Lyapunov exponents of the half-line SHE. arXiv:2007.10212v1, 2020.

[MT59] J. B. McLeod and E. C. Titchmarsh. On the asymptotic distribution of eigenvalues. Quart. J. Math. Oxford Ser. (2), 10:313-320, 1959.

[Mue91] C. Mueller. On the support of solutions to the heat equation with noise. Stochastics, 37:225-245, 1991.

[Nis] NIST Digital Library of Mathematical Functions. http://dlmf.nist.gov/, Release 1.1.0 of 2020-12-15. F. W. J. Olver, A. B. Olde Daalhuis, D. W. Lozier, B. I. Schneider, R. F. Boisvert, C. W. Clark, B. R. Miller, B. V. Saunders, H. S. Cohl, and M. A. McClain, eds.

[Par19a] S. Parekh. The KPZ limit of ASEP with boundary. Commun. Math. Phys., 365(2):569-649, 2019.

[Par19b] S. Parekh. Positive random walks and an identity for half-space SPDE's. arXiv:1901.09449, 2019.

[QS15] Jeremy Quastel and Herbert Spohn. The One-Dimensional KPZ Equation and Its Universality Class. Journal of Statistical Physics, 160(4):965-984, 2015.

[Qua11] Jeremy Quastel. Introduction to KPZ. Current Developments in Mathematics, 2011(1):125-194, 2011.

[Rai00] E. Rains. Correlation functions for symmetrized increasing subsequences. arXiv:0006097, 2000.

[RRV11] J. Ramirez, B. Rider, and B. Viràg. Beta ensembles, stochastic Airy spectrum, and a diffusion. J. Amer. Math. Soc., 24(4):919-944, 2011.

[Sas16] Tomohiro Sasamoto. The 1D Kardar-Parisi-Zhang equation: Height distribution and universality. Progress of Theoretical and Experimental Physics, 2016(2), 02 2016. 022A01.

[SMP17] P. Sasorov, B. Meerson, and S. Prolhac. Large deviations of surface height in the $1+1$-dimensional Kardar-Parisi-Zhang equation: exact long-time results for $\lambda h<0$. J. Stat. Mech., 2017(6):063203, 2017.

[Sos00] A. B. Soshnikov. Gaussian fluctuation for the number of particles in Airy, Bessel, sine, and other determinantal random point fields. J. Stat. Phys., 100(3-4):491-522, 2000.

[SS10] T. Sasamoto and H. Spohn. One-dimensional Kardar-Parisi-Zhang equation: An exact solution and its universality. Phys. Rev. Lett., 104(23), 2010.

[Tak18] K. A. Takeuchi. An appetizer to modern developments on the Kardar-Parisi-Zhang universality class. Physica A: Statistical Mechanics and its Applications, 504(C):77-105, 2018.

[Tsa18] L.-C. Tsai. Exact lower tail large deviations of the KPZ equation. arXiv:1809.03410, 2018.

[TW96] C. A. Tracy and H. Widom. On orthogonal and symplectic matrix ensembles. Commun. Math. Phys, 177(3):727-754, 1996.

[Vir14] B. Virág. Operator limits of random matrices. ICM Proceedings 2014, 4, 2014.

[Wu18] X. Wu. Intermediate disorder regime for half-space directed polymers, 2018.

[Zho20] C. Zhong. Large deviation bounds for the Airy point process. arXiv:1910.00797v5, 2020.

[Zyg18] N. Zygouras. Some algebraic structures in the KPZ universality. arXiv:1812.07204v2, 2018.

Y. H. KIM

Courant Institute, New York University, 251 Mercer Street, New York, NY 10012, USA.

Email address: yujin.kim@cims.nyu.edu 